\title{
3D printing for ecology and evolution: a hands-on guide to turn an idea into reality
}

\section{Nicolas Schtickzelle, Estelle Laurent, Thibaut Morel-Journel}

Université catholique de Louvain

Earth and Life Institute \&Biodiversity Research Centre

Croix du Sud 4, L7.07.04

1348 Louvain-la-Neuve

Belgium

\section{Corresponding author:}

Nicolas Schtickzelle

Nicolas.schtickzelle@uclouvain.be

$+3210472052$

\section{Key-words}

3D printing, 3D scanning, customized ecological objects, methods, stereolithography, open-source lab

\section{Abstract}

3D printing is described as the third industrial revolution: its impact is global in industry and progresses every day in society. It presents a huge potential for ecology and evolution, sciences with a long tradition of inventing and creating objects for research, education and outreach. Its general principle as an additive manufacturing technique is relatively easy to understand: objects are created by adding material layers on top of each other. Although this may seem very straightforward on paper, it is much harder in the real world. Specific knowledge is indeed needed to successfully turn an idea into a real object, because of technical choices and limitations at each step of the implementation. This article aims at helping scientists to jump in the 3D printing revolution, by offering a hands-on guide to current 3D printing technology. We first give a brief overview of uses of 3D printing in ecology and evolution, then review the whole process of object creation, split into three steps: (1) obtaining the digital 3D model of the object of interest, (2) choosing the 3D printing technology and material best adapted to the requirements of its intended use, (3) pre- and post- 
processing the 3D object. We compare the main technologies available and their pros and cons according to the features and the use of the object to be printed. We give specific and key details in appendices, based on examples in ecology and evolution. 


\section{Introduction}

Ecologists and evolutionary biologists have a long tradition of inventing and creating objects for research, education and outreach. The key shared feature of many of these items is that they are highly specific and customized to a very specific application, which originates from the huge variation in model systems used in ecology and evolution, in the field or in the lab. Do-it-yourself and crafting skills are then an important part of the ecologist toolbox. Besides all the traditional manufacturing technologies, a relative newcomer is likely to become a key of this toolbox: three-dimensional (3D) printing.

$3 \mathrm{D}$ printing has received unprecedented attention in the recent years, being described more than once as an industrial revolution (Berman, 2012; Prince, 2014; Barnatt, 2016). Its impact is global, spanning from aerospace engineering (Joshi \& Sheikh, 2015) to dentistry (Dawood, Marti, Sauret-Jackson, \& Darwood, 2015) or chemistry (Ambrosi \& Pumera, 2016; Parra-Cabrera, Achille, Kuhn, \& Ameloot, 2018), and is especially notable in medicine, where it has opened exciting perspectives for the production of bio-printed tissues and organs (e.g. Bose, Vahabzadeh, \& Bandyopadhyay, 2013; Murphy \& Atala, 2014; Patra \& Young, 2016). The widespread appeal of 3D printing in various fields notably resides in its novelty as a fabrication method. It is an additive manufacturing process, which creates 3D objects by joining materials layer upon layer (Guo \& Leu, 2013; Redwood, Schöffer, \& Garret, 2017) (Figure 1), and proved to be especially efficient to produce complex objects (Guo \& Leu, 2013), with lower costs in energy and material (Ambrosi \& Pumera, 2016). The use of additive manufacturing as a complement to traditional subtractive and formative methods (Figure 1) already had a significant economic impact on the industry, which is expected to increase further in the future (Weller, Kleer, \& Piller, 2015).

Yet, the technical innovation is only a part of the explanation of the current enthusiasm for 3D printing. Indeed, the technology itself dates back to the 1980s, when Charles Hull patented the first stereolithography apparatus (SLA), which started the development of 3D printing in the industry (Hull, 1986). The current boom in the 3D-printing world started in the 2000s, with the development of free and open-source printing. A major actor of this democratization of the technology has been the RepRap project (Replicating Rapid prototype, in our context a 3D printer that can print most of its own parts, making it cheaper: Jones et al., 2011), which promotes open-source printing methods, with the objective of creating selfreplicating manufacturing machines. Thereafter, the open-source and do-it-yourself culture has become an integral part of 3D printing (Baden et al., 2015; Mota, 2011; Pearce, 2014). Today, several 3D printing technologies are available at a reasonable cost and with more and more user-friendly interfaces. The technology is mature enough for widespread use for research, education and outreach in multiple fields (Lücking, Sambale, Beutel, \& Scheper, 2015; Ambrosi \& Pumera, 2016; Patra \& Young, 2016). However, the use of 3D printing still remains relatively rare in biology, and ecology and evolutionary biology makes no exception (but see Behm, Waite, Hsieh, \& Helmus, 2018; Walker \& Humphries, 2019). 
Additive manufacturing
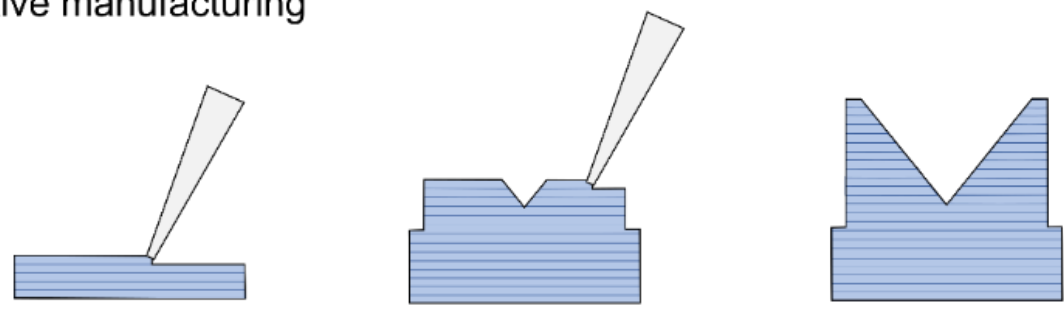

Subtractive manufacturing
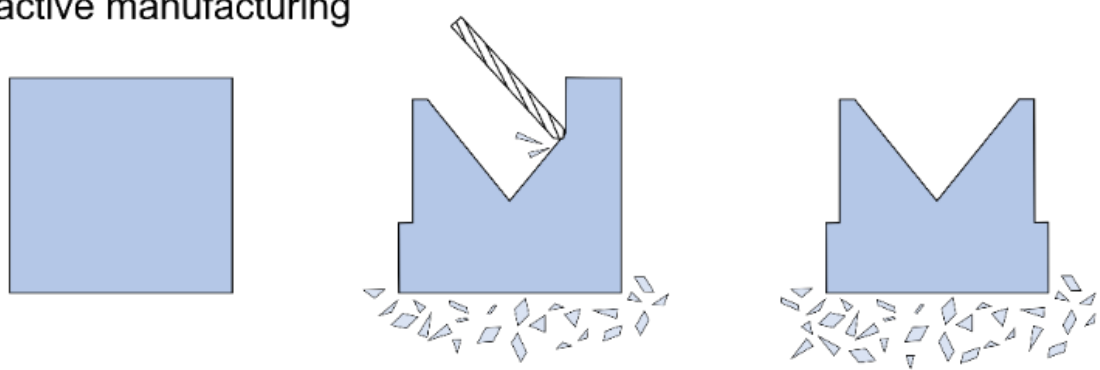

Formative manufacturing
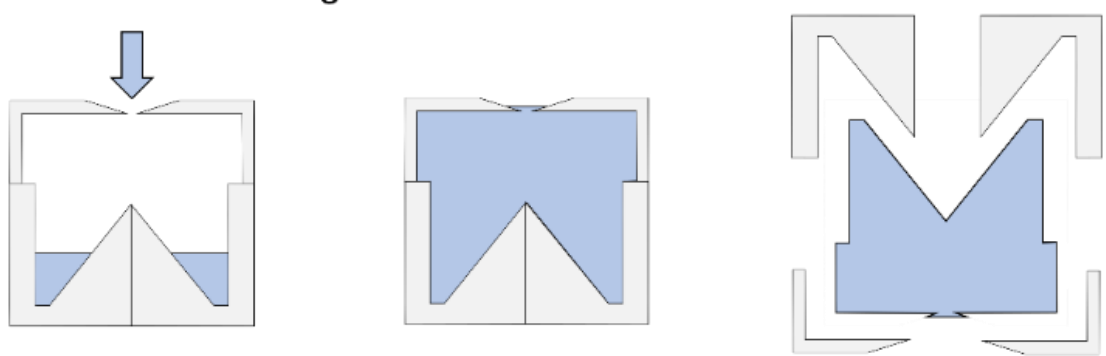

Figure 1. 3D printing is an additive manufacturing technique, building the object layer after layer, which complements classical subtractive manufacturing (objects are created by removing material, e.g. by drilling, milling, sawing or broaching) and formative (objects are formed using heat and pressure, e.g. mould casting).

The general principle of 3D printing is relatively easy to understand: the desired 3D shape is built by superposing layers of material one on top of another (Figure 1). However reliably turning the object description into a real 3D-printed object requires specific knowledge, because of technical choices and limitations at each step of the implementation. In this article, we aim at helping scientists to grasp the opportunities of 3D printing for their research and education and jump in. We start with a quick overview of examples of 3D printing uses in ecology and evolution to illustrate the variety of objects, uses and questions they may help to solve and inspire the reader. We then review the process of object creation using 3D printing technology, split into three steps:

(1) obtaining the digital 3D model of the object of interest,

(2) choosing the 3D printing technology and material,

(3) pre- and post-processing the 3D object.

We compare the main technologies available and their pros and cons according to the features and the use of the object to be printed, complemented by decision keys to help the reader making the best choice for the object to be created. We give very detailed and practical information for all three steps in appendices and when useful refer the reader to additional specialized literature but also to websites and videos existing on the internet, which are 
essential resources to understand and make use of 3D printing. We then use a few of the examples in ecology and evolution to illustrate choices made for their successful implementation. Finally, we conclude with mentioning some current developments of the 3D printing technology that are likely to further expand the possibilities soon.

\section{A brief overview of 3D printing use in ecology and evolution}

3D printing has opened a world of possibilities for the design and creation of customized objects. Recent reviews offer a relatively comprehensive synthesis of the current use of 3D printed objects in ecology and evolution (Behm et al., 2018; Walker \& Humphries, 2019). Here, we give a brief overview of the most frequent applications, grouped into three main categories (Figure 2): replicas of biological material, technical tools, and customized experimental devices.

3D printing was prominently used to create replicas of biological material, which are frequently used in ecological research. For instance, in thermal ecology, 3D printed lizard models were used as "more standard, lighter, durable, and inexpensive" alternatives to the traditional copper hollow electroformed models for field studies about their thermal properties (Watson \& Francis, 2015). In another study, 3D printed lizards were also found to be more efficient than clay models in studies of predation rate (Behm et al., 2018) (Figure 2A). In functional ecology, key questions often involve studying the impact of specific morphological properties of the organism (or part of it). For example, heads of bat species were scanned and 3D printed for their aerodynamical properties to be tested in a wind tunnel (Vanderelst, Peremans, Razak, Verstraelen, \& Dimitriadis, 2015); similarly, 3D printed beaks of kingfishers were used to study the evolution of drag reduction at the air-water interface (Crandell, Howe, \& Falkingham, 2019). 3D printing furthermore allows to go one step further than replicating biological material: creating highly controlled variants in order to manipulate morphological features independently. For instance, 3D printed moulds were used to cast realistic flowers in silicone in a study aiming at disentangling visual and olfactory signals in orchids, by creating chimeras combining scent-free silicone flower parts with real floral organs (Policha et al., 2016) (Figure 2B). Other examples include 3D printed flower corollas of specific mathematically characterized shapes to test the foraging behaviour of pollinators (Campos, Bradshaw, \& Daniel, 2015) (Figure 2C), or eggs to study egg rejection among robins (Igic et al., 2015) (Figure 2D). Replicas of biological material are also very useful for research and education based on museum specimens, as exemplified with virtual skeletons (Niven, Steele, Finke, Gernat, \& Hublin, 2009; Mitsopoulou et al., 2015), where scanning and 3D printing biological replicas allows much faster and safer sharing of such specimens and even the creation of missing bones based on mathematical methods and allometric analyses (Figure $2 \mathrm{E}$ ). Finally, highly detailed 3D printed replicas of organisms are also used in outreach activities to allow blind people to discover insect diversity through touching (Laurentino, 2019) (Figure 2F).

Besides biological models, 3D printing enables the creation of highly customized experimental devices, and therefore the design of experiments that would be prohibitively 
challenging without this technology. For our own research, we created artificial landscape microcosms to perform experimental studies of landscape configuration and connectivity on protist (meta)population dynamics (Morel Journel \& Schtickzelle, in prep.) (Figure 2G). Other recent examples are emergence traps of ambrosia beetles (Berry et al., 2016) (Figure 2H), fish graders to quickly sort zebrafish by width (O'Reilly-Pol, Kniepkamp, \& Johnson, 2017) (Figure 21), a free-swimming soft robotic jellyfish aimed at exploring coral reefs (Frame, Lopez, Curet, \& Engeberg, 2018) (Figure 2J), a flying drone to collect observational data and biological samples from living whales (Bennett et al., 2015) (Figure 2K), a fully controllable robot inspired by a natural aquatic predator to study anxiety in the zebrafish animal model (Cianca, Bartolini, Porfiri, \& Macrì, 2013) (Figure 2L), and a soft robotic arm for delicate deep-sea biological exploration (Phillips et al., 2018) (Figure 2M).

Finally, 3D printing is also very efficient in producing various tools to be used in the field or in the lab. In particular, it can produce cheaper, and sometimes more reliable, alternative to experimental tools classically used in ecology, such as micropipettes (Baden et al., 2015) (Figure $2 \mathrm{~N}$ ), or a bench centrifuge for microtubes (Cathalgarvey, 2009) (Figure 20). Lücking et al. (2015) present an array of frequently used labware for biological research that could be created with the current generations of materials currently used for 3D printers. Potential applications are even more numerous when considering that 3D printing is part of the larger "maker movement" relying on Do-It-Yourself methods. For example, it can combine with electronics (such as open source Arduino: www.arduino.cc) to create scientific equipment and devices (Pearce, 2014; Baden et al., 2015), for example a bench shaker/rocker (akshay_d21, 2018) (Figure 2P). When used routinely in laboratories, 3D printing could significantly reduce research costs on the long run, also by facilitating repairs of scientific equipment or allowing the production of consumables (Canessa, Fonda, \& Zennaro, 2013). If this possibility would directly benefit the whole scientific community, it could be a game changer for the scientists working in developing countries, who are the most impacted by the financial and logistic challenges of acquiring the top-end equipment required to conduct innovative research (Canessa et al., 2013). 


\section{Replicas of biological material}
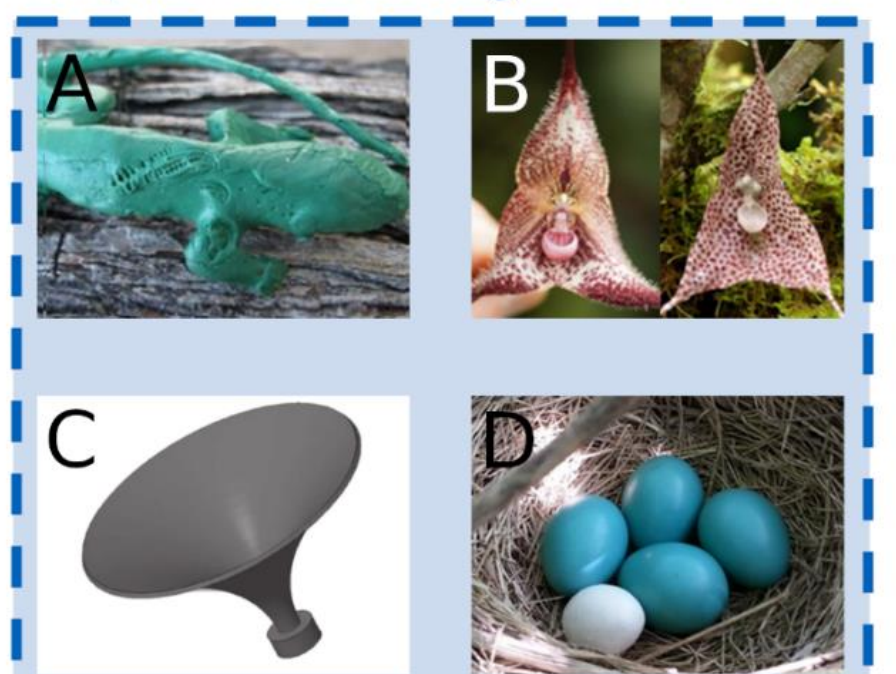

Customized experimental material
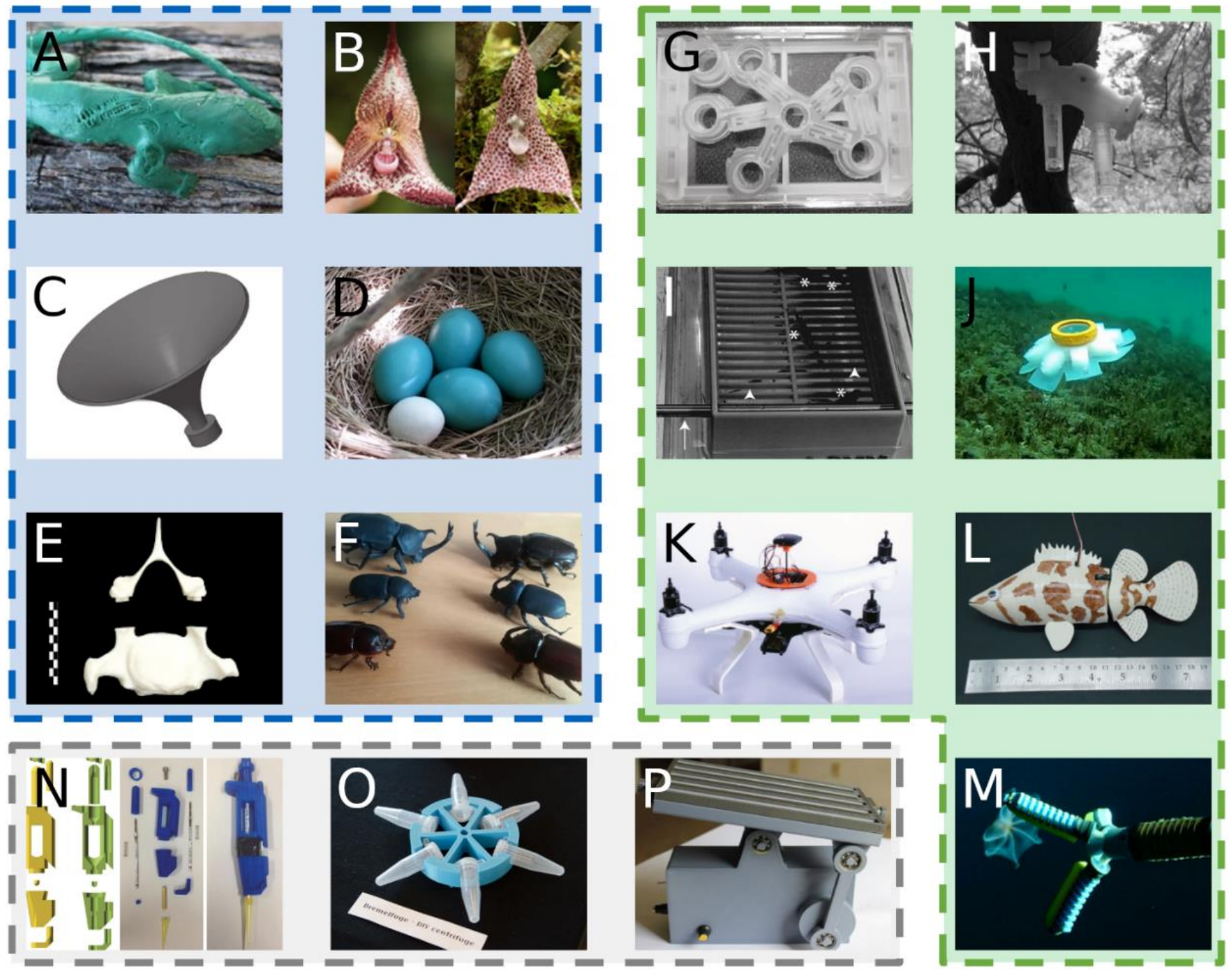

Technical tools

Figure 2. Examples of use of 3D printed objects in ecological and evolutionary research and education. Replicas of biological material, such as (A) lizard models to study predation rate (Behm et al., 2018), (B) scent-free flowers to disentangle visual and olfactory cues produced by orchids (Policha et al., 2016), (C) flower corollas of specific mathematically characterized shapes to test the foraging behaviour of pollinators (Campos et al., 2015), (D) eggs to study egg rejection among robins (Igic et al., 2015), (E) cervical vertebra of the dwarf elephant Palaeoloxodon tiliensis (Mitsopoulou et al., 2015), and (F) insects used in outreach activities targeted at blind people (Laurentino, 2019). Customized experimental material to be used in the field or in the lab, such as (G) artificial landscape microcosms to study protist (meta)population dynamics (Morel Journel \& Schtickzelle, in prep.), (H) emergence traps of ambrosia beetles (Berry et al., 2016), (I) fish graders to quickly sort zebrafish by width (O'Reilly-Pol et al., 2017), (J) a free-swimming soft robotic jellyfish aimed at exploring coral reefs (Frame et al., 2018), (K) a flying drone to collect observational data and biological samples from living whales (Bennett et al., 2015), (L) a fully controllable robot inspired by a natural aquatic predator to study anxiety in the zebrafish animal model (Cianca et al., 2013), and (M) a soft robotic arm for delicate deep-sea biological exploration (Phillips et al., 2018). Technical tools, such as (N) a low cost open source micropipette (Baden et al., 2015), (O) the Dremelfuge, a microtube rotor to transform a small drill into a lab centrifuge (Cathalgarvey, 2009), and (P) a low cost open source laboratory rocker (akshay_d21, 2018). 


\section{Step 1: obtaining a digital 3D model of the object}

The first step in a 3D printing project is to acquire the digital counterpart of the object to be printed. A digital 3D model is a geometric description of the object volume in the form of a polygonal 3D mesh of points (called vertices) connected by lines (called segments or edges); polygons are flat surfaces and curved surfaces are approximated by using a high density of smaller polygons. There are three options to obtain the digital 3D model of the object to be printed:

(1) finding an existing digital 3D model,

(2) scanning an existing real object, or

(3) creating the digital 3D model from scratch using specific software.

In Figure 3, we present a decision tree to help choosing the best option depending on features of the desired object. It is also possible to combine these approaches by modifying an existing digital 3D model or a model obtained from 3D scanning. As an alternative, professional companies (e.g. Volumatik, www.volumatik.com, or Shapeways, www.shapeways.com) and marketplaces (Table S2) can create models on-demand from your own description or design.

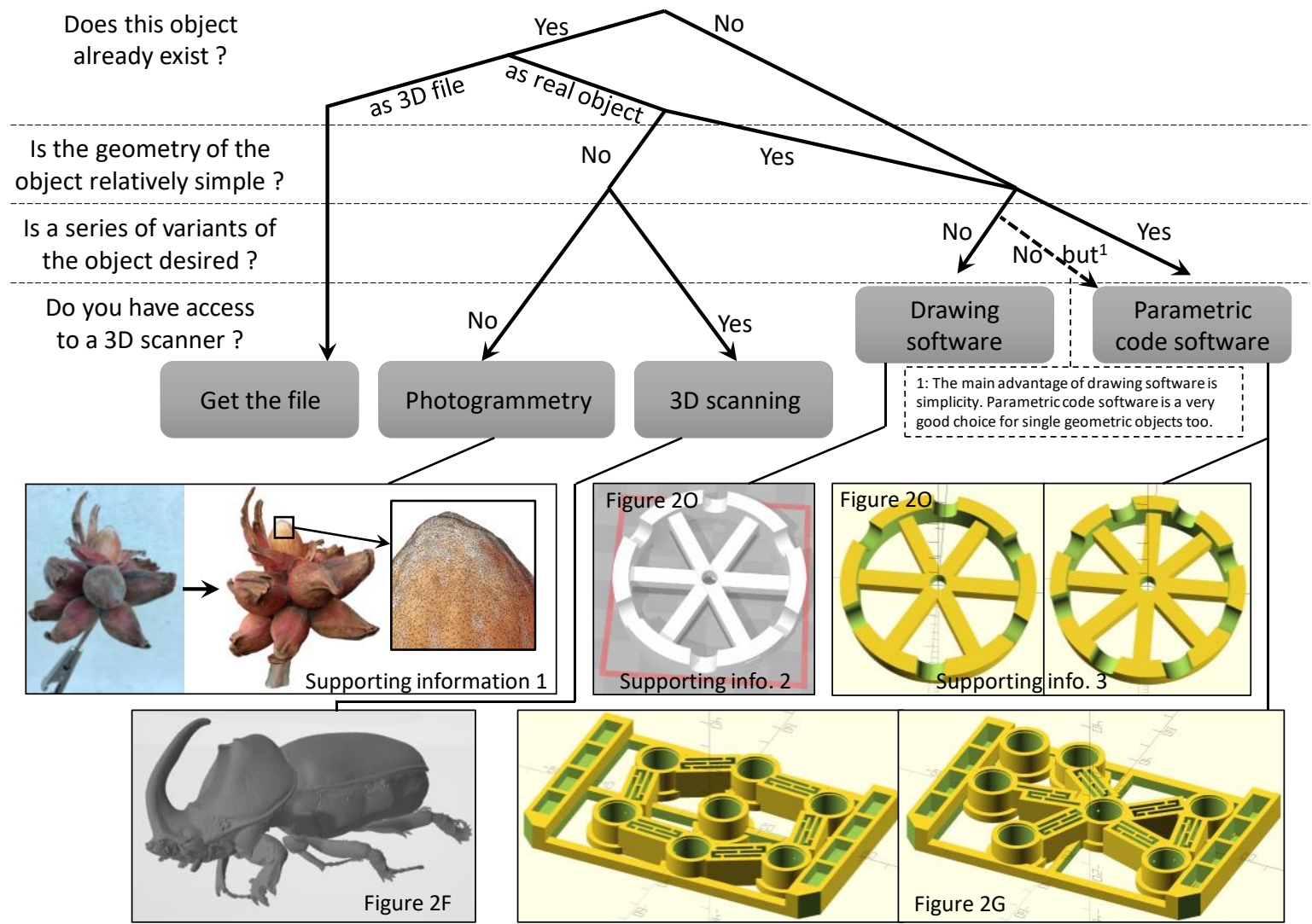

Figure 3. Decision tree to determine the best approach to obtain the digital 3D model of the object of interest: getting the digital 3D model file of an existing object, scanning an existing and complex object such as biological material, or creating it from scratch using drawing software or parametric code software. See Appendices (1 to 3) for details on the creation steps of the corresponding examples, extra information and related files. 


\section{Finding an existing digital 3D model}

When it is available, the most immediate approach is to retrieve the file corresponding to an existing digital 3D model (see e.g. examples in Figure 2N, O \& P). Table S1 lists several major sources for finding (free or paid) digital 3D models online, some with detailed instructions for print and assembly. The catalogue of digital 3D model online sources is likely to evolve rapidly given the highly dynamic nature of 3D printing community and industry; e.g. Github (github.com), one of the reference repositories for computer codes, recently added a 3D model rendering facility, attracting digital 3D models. Therefore, searching on major internet search engines with keys such as "stl" (one of the classical file types for 3D digital models), "3D model", "3D print" and keywords describing your object of interest should be considered as an efficient additional source to find digital 3D models online.

\section{Scanning an existing real object}

Very often, the object to be printed does not already exist in the form of a digital 3D model, but exists as a real object, such as a biological organism or element that one wants to reproduce (and possibly modify). 3D scanning techniques can be used to create its digital 3D model counterpart (see e.g. examples in Figure 2A, B, E \& F), using a scanner or a camera (photogrammetry).

In the scanner approach, 3D scanners cover the whole volume of the object with a 3D mesh of individual point locations acquired one after the other. A turntable must be used to rotate the object in front of the scanner, or the scanner itself must rotate around the object. The position of each location in space is deduced either from a probe that physically touches the object (contact-based scanner), through a laser dot projected on the object (laser-based scanner) or through the distortion of a light pattern (generally parallel stripes) projected on the object (structured light scanner). Contact-based scanners are mainly industry devices, while a large range of all-in-one devices exist for laser-based and structured light scanners, for all needs and budget. Listing them is out of the scope of the present paper but searching the internet with the "3D scanner" key will return enough hits to look for the appropriate solution for your needs; websites also exist that list and compare 3D scanners (as well as 3D printers) (e.g. Aniwaa, https://www.aniwaa.com/).

In the photogrammetry approach, a series of pictures of the object are shot from various directions and stitched together based on the change in the relative position of landmarks between pictures. This method best works with objects presenting a heterogeneous surface (Bracer, 2017), which is the case of many biological material elements. New photogrammetry applications are developed to reconstruct digital 3D models from a series of pictures shot by a camera or a smartphone (Prusa, 2018), with increasingly better results. Open source solutions exist but they are not always user-friendly. often not easy to use (e.g. Falkingham, 2012). We found Recap Photo from Autodesk, a leader in Computer Aided Design (CAD) that offers a special free education license (https://www.autodesk.com/education/home), quite efficient in reconstructing a 3D object from a series of photos shot with a smartphone (Figure 3; Appendix 1). Given how widespread smartphones are nowadays, equipped with more and 
more powerful cameras, this approach might often be a very affordable and efficient mean of scanning a real object into a digital 3D model.

\section{Designing the model using software}

The third option to obtain a digital 3D model of the object of interest is to create it from scratch, using specialized CAD (Computer Aided Design) software; this is best done when the geometry of the object is relatively simple (see e.g. examples in Figure 2C, D, G, I \& O). Two approaches exist: (1) using a drawing software, where the object is created by drawing it directly in 3D, and (2) using a parametric code software, where the object is created from a code/script that defines its shape in space (Figure 3; see Appendices $2 \& 3$ for detailed illustration of how to create the microtube rotor, Figure $2 \mathrm{G}$, using each of these approaches). The general principle is the same in both approaches: the object is created by a series of shapes (cube, sphere, cylinder, polyhedron, and their variants...) with specific dimensions that are translated and/or rotated to be placed at a precise position in space; shapes combine into the object, and negative shapes are used to create holes.

Drawing software solutions are more visual and require much less prior knowledge than parametric code software as the result can be viewed directly on screen. The recent boom of 3D printing led major actors to develop user-friendly and relatively easy to master drawing CAD software. To cite only a few (more are reviewed in Redwood et al., 2017): Tinkercad offers free online CAD software and tutorials for beginners (www.tinkercad.com/learn), and Microsoft has developed a free 3D drawing CAD application called 3D Builder (available in Windows store). Yet, code-based 3D model design likely offers more versatility than a drawing software. Its parametric nature is a main advantage to create tools and models for experiments: variants of the same object can be easily created from the same code, only by changing the values of the parameters (Figure 3). In this context, OpenSCAD (www.openscad.org) is a masterpiece: it is free, multiplatform, open source and has a very detailed online documentation and an active community, meaning that users can reuse existing code portions to greatly simplify the creation of their own model. Such code portions can be found e.g. in the MCAD library (https://github.com/openscad/MCAD) or Thingiverse (https://www.thingiverse.com). We used OpenSCAD to produce landscape configuration variants of our microcosms for protists (Figure 3 ). 


\section{Step 2: Choosing the optimal printing technology and material}

There are several 3D printing technologies (Figure 4): (material extrusion, photopolymerization, and powder-based printing), each with a specific range of materials associated to physical features such as resistance, heat tolerance, or ability to print hollow or watertight objects. Biocompatible applications are also possible (Lücking, Sambale, Schnaars, et al., 2015; Oskui et al., 2016). Each technology and material present their own pros and cons (Table 1), and the key to successful 3D printing is to choose the best technology-material couple according to the object nature and future use. This requires some knowledge of their features and limitations to take them into account already when designing the digital 3D model. Indeed, anything can be drawn as a digital 3D model, but not everything can be reliably 3D printed, and limitations depend on the specific technology-printer-material association (e.g. Redwood et al., 2017).

Some of these technologies and materials are available for consumer 3D printers and some are still reserved to professional/industrial use due to their price (sometimes $>100.000$ $€ / \$)$ and the expertise required (Table 1). Nevertheless, there is a growing number of companies offering on-demand 3D printing service (see Table S2 for examples) and price comparison services (e.g. All3DP, all3dp.com). Fablabs (Fabrication laboratories) are also rapidly developing; they are collaborative workshops allowing access to (basic) 3D printers (as well as other tools such as laser cutters, milling machines...); there may be one in your university or city, often referenced on the internet.
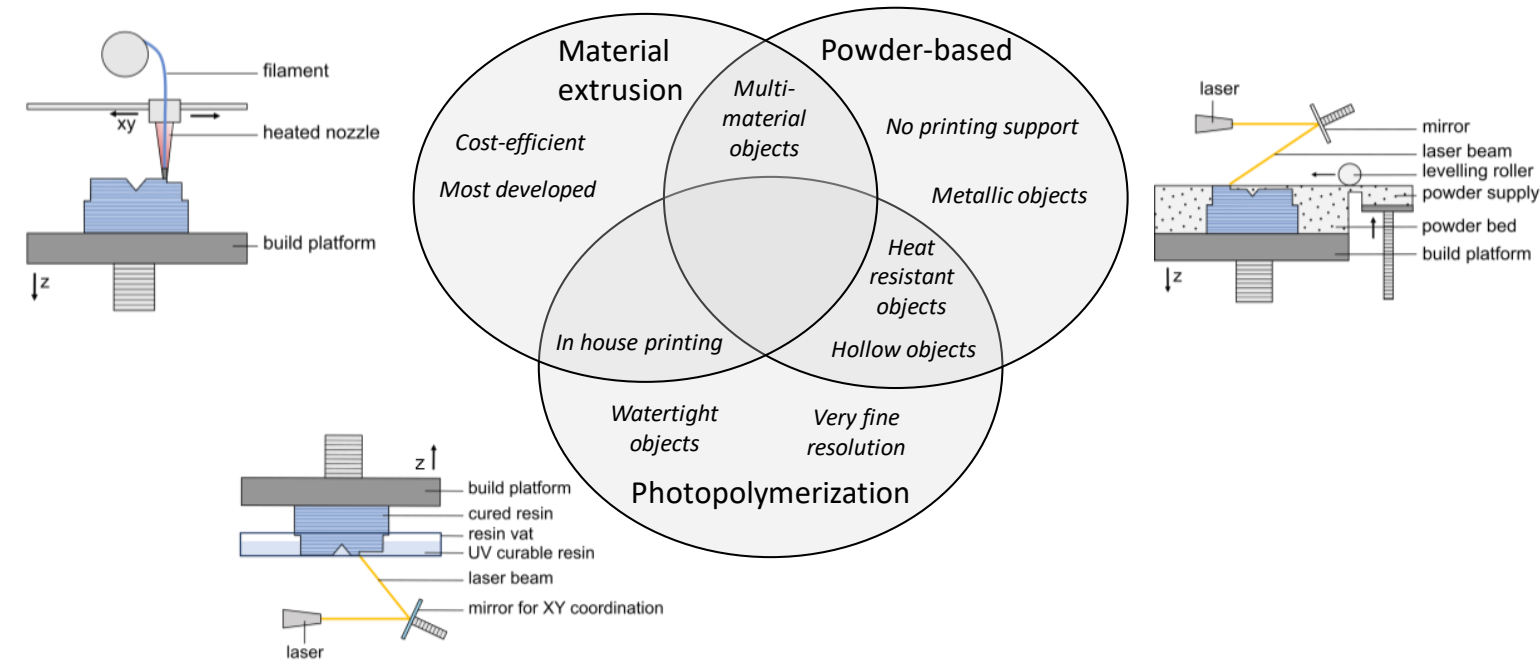

Figure 4. The main 3D printing technologies use the same approach: 3D objects are created by joining materials layer upon layer (z dimension). Material extrusion technologies extrude material from a moving printhead. Photopolymerization technologies use a source of light to polymerize a liquid resin. Powder-based technologies bind particles together either by heat fusion or by extruding a binding agent from a moving nozzle. 


\begin{tabular}{|c|c|c|c|}
\hline & Material extrusion & Photopolymerization & Powder-based \\
\hline $\begin{array}{l}\text { Accessibility (printer } \\
\text { price and expertise } \\
\text { needed) }+\end{array}$ & $\begin{array}{l}\text { FFF: Consumer, prosumer, professional } \\
(100->250.000 €) \\
\text { Material Jetting: professional (10.000-100.000€) }\end{array}$ & $\begin{array}{l}\text { Prosumer, professional } \quad \text { (500- } \\
250.000 €)\end{array}$ & Professional (5000-250.000€) \\
\hline Cost of printing $\ddagger$ & $\begin{array}{l}\text { FFF: } 81 €(1 €) \\
\text { Material Jetting: } 65-83 €\end{array}$ & $131 €(24 €)$ & $\begin{array}{l}\text { SLS: } 13 € \\
\text { SLM: } 114 €\end{array}$ \\
\hline $\begin{array}{l}\text { Layer thickness } \\
\text { (Z-axis resolution) }\end{array}$ & $\begin{array}{l}\text { FFF: } 50-400 \mu \mathrm{m} \\
\text { Material Jetting: } 16-30 \mu \mathrm{m}\end{array}$ & $25-100 \mu \mathrm{m}$ & $\begin{array}{l}\text { SLS: } 100 \mu \mathrm{m} \\
\text { SLM: } 50 \mu \mathrm{m}\end{array}$ \\
\hline $\begin{array}{l}\text { Minimal feature size } \\
\text { (X- and Y-axis } \\
\text { resolution) }\end{array}$ & $\begin{array}{l}\text { Positive feature (wall or pin) } \\
\text { FFF: } 0.8 \mathrm{~mm} \\
\text { Material Jetting: } 1 \mathrm{~mm} \\
\text { Negative feature (hole) } \\
\text { FFF: } 2 \mathrm{~mm} \\
\text { Material Jetting: } 0.5 \mathrm{~mm}\end{array}$ & $\begin{array}{l}\text { Positive feature (wall or pin) } \\
0.5 \mathrm{~mm} \\
\text { Negative feature (hole) } \\
0.5 \mathrm{~mm}\end{array}$ & $\begin{array}{l}\text { Positive feature (wall or pin) } \\
\text { SLS: } 0.7 \mathrm{~mm} \\
\text { SLM: } 0.4 \mathrm{~mm} \\
\text { Negative feature (hole) } \\
\text { SLS: } 1.5 \mathrm{~mm} \\
\text { SLM: } 1.5 \mathrm{~mm}\end{array}$ \\
\hline $\begin{array}{l}\text { Supports for overhangs } \\
\text { needed }\end{array}$ & Yes; dissolvable supports possible & $\begin{array}{l}\text { Yes; must be physically detached } \\
\text { in post-processing }\end{array}$ & No \\
\hline Multimaterial possible & Yes & No & In development \\
\hline Hollow objects & Infill usually needed & Material evacuation hole needed & Material evacuation hole needed \\
\hline Watertight objects & No & Yes & Partial \\
\hline
\end{tabular}

Table 1. Features of the main 3D printing technologies.

† Printer prices have been retrieved from the 3D printer comparison at www.aniwaa.com on 23/09/2019.

₹ For comparison, this is the cost of the "features" object (see Appendix 4: $37.5 * 76.2 * 10 \mathrm{~mm}, 11.9 \mathrm{~cm}^{3}$ ), as quoted from Materialise OnSite, one of the leading on-demand 3D printing companies; the price may differ among companies, and price ranking for the technologies may change according to the 3D object to be printed. When possible, an in-house printing price is also mentioned in parentheses, which does not include the cost of the printer nor of the work time, but only the cost of the material used to print the object (Stereolithography on a Formlabs Form1+ printer with clear resin, FFF on a BCN3D Sigma printer with white PLA). 


\section{Material extrusion}

The extrusion of material from a moving printhead started with the Fused Deposition Modelling (FDM) technology, a term trademarked by Stratasys, which belongs to the Fused Filament Fabrication (FFF) technology. FFF is the cheapest and most common 3D printing technology, especially in the consumer and RepRap markets. A plastic filament is fed through a heated printhead, which melts it and extrudes it to create a layer of the desired shape (Figure 4) (Solid Concept, 2013b). The receiving platform is lowered after each layer and the object created layer after layer. Some printers support printing simultaneously with several printheads, each extruding one material, allowing creating multi-coloured objects or objects that require different functional parts; this also permits the use of a soluble material for printing supports (see step 3 below). FFF printers, even low-cost consumer-oriented printers or RepRap printers, allow printing with an extensive range of relatively cheap materials: thermoplastics (e.g. acrylonitrile butadiene styrene, ABS; polylactic acid, PLA), nylon, or rubber-like materials. Another, more recent, material extrusion technology is Material Jetting, the 3D printing counterpart of classical 2D inkjets printers: a moving printhead jets hundreds of tiny droplets of photopolymer and cures them instantly with UV light. A relatively wide range of resins with different properties are available but the high price of the material and the technology currently reserves material jetting to specific applications.

Material extrusion technology is likely the most suitable when the object does not need specific physical properties other than some structural resistance, when cost of printed parts is an important aspect, and/or when in-house printing is desired with limited financial investment. A good example of such a situation is the emergence traps of ambrosia beetles (Berry et al., 2016) (Figure 21), printed in ABS for a $1.30 \$$ cost per piece using a relatively basic FFF printer. Artificial flowers printed in ABS were rigid and odourless, two important features for their use in studying foraging behaviour of pollinators (Campos et al., 2015) (Figure 2C). Many labware are also best printed with material extrusion (e.g. Figure 2N, O \& P).

\section{Photopolymerization}

Photopolymerization techniques print the 3D object by polymerizing a liquid polymer resin using a source of light, either a laser that progressively draws the shape of the layer (in Stereolithography, SLA) or a projector that projects the full image of the layer (in Digital Light Processing, DLP). The object is constructed layer after layer, usually bottom-up, on a platform immerged into a vat containing the resin (Figure 4) (Formlabs, 2017). Photopolymerization techniques produce highly accurate parts with smooth surface finishes owing to their high resolution and because the polymerization in each resin layer is not complete after one light pass, allowing for polymer bounds to create with the next layer. This also makes objects printed with photopolymerization watertight, which makes them excellent for microfluidic systems. Such properties cannot be easily achieved with material extrusion because the plastic layers are not structurally bound together the same way as molecules within a layer are (material extrusion is highly anisotropic). The range of resin material is rapidly expanding, with 
various colours or transparent plastic-type resin, more or less flexible resin, high-temperature or castable resin (e.g. suitable for creating moulds for injection-moulding small and detailed objects, or to create objects that need to be autoclaved for sterility reasons), biocompatible resin (originally made for dentistry but suitable for e.g. microorganism culture vessels), and even ceramic resin that can be fired to create objects resisting high temperature and chemically inert (Eckel et al., 2016).

Photopolymerization is the best choice when water-tightness and/or a high-level of details must be obtained, and when in-house printing is desired for rapid prototyping. This was for example the case for our own artificial landscapes that we use to study dispersal and metapopulations dynamics in protist microcosms (Morel Journel \& Schtickzelle, in prep.) (Figure 2G): these microcosms need to be filled with protist cultures in liquid medium and small ( $<1 \mathrm{~mm}$ diameter) but precisely dimensioned holes must be maintained in some wall to allow protists to disperse through the connecting corridors. Photopolymerization offered us these water-tightness and dimensional accuracy we needed, in addition to the ability to acquire a $3 \mathrm{D}$ printer for a reasonable cost to be able to design and print our microcosms without the delays inherent to printed by an external company. The evALLution replicas of insects were also best printed using photopolymerization because of the high level of details required for the $3 \mathrm{D}$ printed replicas (Figure $2 \mathrm{~F}$ ); in collaboration with the scientists at evALLution, we are currently testing printing these insects using flexible resin to allow better resistance to handling by blind people.

\section{Powder-based techniques}

Powder-based techniques bind powder particles together to create the solid object, layer by layer (Figure 4), either by heat fusion using a laser that melts/sinters the powder particles (Selective Laser Sintering, SLS for plastic-type powder; and Selective Laser Melting, SLM for metal powder), or by extruding a binding agent from a moving nozzle (Binder Jetting). Once a layer of powder has been solidified, the print platform moves down slightly and a roller mechanism spreads a new layer of powder atop the object (Solid Concept, 2013a). The binding of powder particles also happens among layers, as it does with photopolymerization resins, ensuring a better adhesion of the different layers and more isotropic objects compared to those printed with material extrusion. The object is printed top-down and is encased into unmelted powder, which acts as support for the printed object (see step 3). Materials available for SLS printing are mainly nylon polyamides, which can be mixed with another material to achieve specific mechanical properties (e.g. carbon, glass or aluminium enhance heat resistance and stiffness). SLM allows using many industrial metals such as stainless steel, titanium, aluminium, and different alloys. Some of these materials bear properties highly desirable in some areas of ecology and evolution research or education, such as biocompatibility, resistance to autoclave, and resistance to adverse weather conditions. However, powder-based 3D printing technology, especially SLM, is still expensive and reserved for industrial use, although accessible to the customer via professional 3D printing services (Table S2). Binder Jetting is available with sand and metal materials. This technology 
is an efficient and cost-effective way of producing multicolour architecture models, and may reveal the best choice to produce replicas of natural organisms or ecosystems for educational purposes.

Powder-based techniques are the best choice when particular properties are needed for the object that cannot be obtained with material extrusion of photopolymerization, e.g. those associated to metal objects. They are also a must to avoid the need for supports during the printing process (see step 3 ) when these have adverse consequences for the final result. However, the technology is still very expensive, both for the printer acquisition and for the materials (Figure 1). It is currently accessible through ordering prints from a 3D printing company (Table S2), but this may imply non-negligible cost and delivery delay. These limitations might explain why none of the examples we cite in ecology and evolution uses powder-based techniques.

\section{Step 3: pre- and post-processing the 3D object}

Before it can be printed, a digital 3D model must be prepared and optimized. This step is crucial to ensure reliable printing, and a good understanding of how the 3D printing process works is important, both in general and for the specific technology and printer chosen for the object. There are three main aspects to consider. First, support structures may need to be added to the object printed with material extrusion and photopolymerization to counterbalance the effect of the forces acting on the object during printing (gravity, peeling process, temperature and/or curing stresses). Second, printing enclosed volumes (i.e. empty cavities) needs supports but also an escape hole to let the resin (photopolymerization) or powder (powder-based printing) escape after printing and relieve suction forces due to the peeling process in photopolymerization. Third, 3D printing technologies and the object they print are not isotropic, i.e. the printing orientation will affect the printed object. More details and examples of these pre-processing steps and their importance are given in Appendix 4. Detailed information on how to best design, print and post-process 3D objects with a specific technology can be found in "The 3D Printing Handbook" (Redwood et al., 2017), one of the best, though accessible to non-specialists, syntheses at the time of writing.

Sending the digital 3D model of the object to the printer often does not require complex steps. Many printers come with a dedicated software that will take care of steps such as best orienting the object and adding supports, but also repairing minor problems with the digital $3 \mathrm{D}$ model and slicing the objects in the layers that will be printed one after the other. However, with some RepRap printers, these steps may need to be performed by the user before sending the digital 3D model to the printer (see Appendix 5).

Once the 3D printing of the object is finished, the object is rarely ready for use. The amount of post-processing needed depends on the printing technology used and the use of the object, which may require changing the appearance or mechanical properties of the object. Supports need to be removed, the surface may need polishing or can be painted. The variety 
of such treatments is too large to offer a complete review here, but we discuss and illustrate a few important points in Appendix 6.

\section{Conclusions and perspectives}

3D printing technology, the third industrial revolution, is currently at an "adaptive radiation" stage of its history. On the one hand, many technologies are already ready for use, which has created an ecosystem with a nearly infinite number of possibilities for their use. We believe that 3D printing has now reached a state of maturity where its use in ecology and evolution will flourish for different applications: one-time cases in the framework of a specific experiment or course, sustained uses to experimental devices tailored to very specific aims, but also to produce generic consumables and (parts for) equipment to save the costs on research and education. This guide presents an overview of the 3D-printing technologies and methods already available for researches, but new developments and improvements in 3D printing technologies and materials are continuously made, opening new possibilities. As a matter of examples, we can cite (1) the development of Continuous Liquid Interface Production (CLIP) technology (Tumbleston et al., 2015), a Digital Light Processing photopolymerization technology developed by Carbon3D (carbon3d.com) tens of times faster than current technique, which uses a dead zone in the resin vat to build the object continuously without the need for a peeling process, producing objects that are isotropic; (2) the extension of Fused Filament Fabrication to glass (Klein et al., 2015) that could be very useful in laboratory ecology and evolution; or (3) the use of Computational Hydrographic Printing to affix a complex colour pattern on a 3D object (formlabs.com/blog/full-colorpattern-3d-prints-computational-hydrographics/), a must to create realistic colour biological replicas. Many other developments should be expected, making 3D printing a real manufacturing revolution, also for scientists.

\section{Acknowledgments}

NS is Senior Research Associate of the F.R.S.-FNRS. EL is teaching assistant at UCLouvain. TMJ was supported by a Move-In-Louvain Marie Curie Action postdoctoral fellowship. This work was financially supported by UCLouvain (ARC 18-23/095) and F.R.S.-FNRS (PDR T.0211.19). We thank Viktoriia Radchuk, Guillaume Le Goff, Gavin Thomas and an anonymous reviewer for comments on previous drafts of this manuscript. This work is contribution BRCXXX of the Biodiversity Research Centre at UCLouvain. 


\section{Author contributions}

NS designed the project, structured the review and led the writing of the manuscript. TMJ drew the first version of several figures. All authors contributed critically to the drafts and gave final approval for publication.

\section{Disclaimer}

Due to the nature of 3D printing technology, many pieces of information and software cited here are linked to commercial companies that make a business of it. We state that we do not have any link with, or take any benefit from, any of these companies. References herein to any specific commercial product, process, or service by trade name, trademark, manufacturer, or otherwise, does not necessarily constitute or imply its endorsement or recommendation; such references are made only for identification and explanation without intent to infringe. 


\section{References}

akshay_d21. (2018). Open Source Laboratory Rocker / Mixer / Shaker (Lab Rocker). Retrieved 4 April 2019, from https://www.thingiverse.com/thing:2948680

Ambrosi, A., \& Pumera, M. (2016). 3D-printing technologies for electrochemical applications. Chemical Society Reviews, 45(10), 2740-2755.

Baden, T., Chagas, A. M., Gage, G., Marzullo, T., Prieto-Godino, L. L., \& Euler, T. (2015). Open Labware: 3-D Printing Your Own Lab Equipment. PLOS Biology, 13(3), e1002086. doi:10.1371/journal.pbio.1002086

Barnatt, C. (2016). 3D Printing: Third Edition. CreateSpace Independent Publishing Platform.

Behm, J. E., Waite, B. R., Hsieh, S. T., \& Helmus, M. R. (2018). Benefits and limitations of threedimensional printing technology for ecological research. BMC Ecology, 18(1), 32. doi:10.1186/s12898-018-0190-z

Bennett, A., Barrett, D., Preston, V., Woo, J., Chandra, S., Diggins, D., ... Kerr, I. (2015). Autonomous vehicles for remote sample collection: Enabling marine research. In OCEANS 2015 - Genova (pp. 1-8). doi:10.1109/OCEANS-Genova.2015.7271621

Berman, B. (2012). 3-D printing: The new industrial revolution. Business Horizons, 55(2), 155162. doi:https://doi.org/10.1016/j.bushor.2011.11.003

Berry, D., Selby, R. D., Horvath, J. C., Cameron, R. H., Porqueras, D., \& Stouthamer, R. (2016). A Modular System of 3D Printed Emergence Traps for Studying the Biology of Shot Hole Borers and Other Scolytinae. Journal of Economic Entomology, 109(2), 969-972. doi:10.1093/jee/tov407

Bose, S., Vahabzadeh, S., \& Bandyopadhyay, A. (2013). Bone tissue engineering using 3D printing. Materials Today, 16(12), 496-504.

Bracer, J. (2017, April 15). Photogrammetry vs 3D Scanner. Retrieved 21 September 2018, from https://www.youtube.com/watch?v=20jvnEtgRIU

Campos, E. O., Bradshaw, H. D., \& Daniel, T. L. (2015). Shape matters: corolla curvature improves nectar discovery in the hawkmoth Manduca sexta. Functional Ecology, 29(4), 462-468. doi:10.1111/1365-2435.12378

Canessa, E., Fonda, C., \& Zennaro, M. (2013). Low-cost 3D Printing for Science, Education and Sustainable Development. ITCP - The Abdus Salam International Centre for Theoretical Physics. Retrieved from http://web.archive.org/web/20150402165048/http://sdu.ictp.it/3d/book.html

Cathalgarvey. (2009, December 23). DremelFuge - A One-Piece Centrifuge for Rotary Tools by cathalgarvey. $\quad$ Retrieved 24 September 2018, from https://www.thingiverse.com/thing:1483

Cianca, V., Bartolini, T., Porfiri, M., \& Macrì, S. (2013). A Robotics-Based Behavioral Paradigm to Measure Anxiety-Related Responses in Zebrafish. PLOS ONE, 8(7), e69661. doi:10.1371/journal.pone.0069661

Crandell, K. E., Howe, R. O., \& Falkingham, P. L. (2019). Repeated evolution of drag reduction at the air-water interface in diving kingfishers. Journal of the Royal Society Interface. doi:10.1098/rsif.2019.0125

Dawood, A., Marti, B. M., Sauret-Jackson, V., \& Darwood, A. (2015). 3D printing in dentistry. British Dental Journal, 219(11), 521. 
Eckel, Z. C., Zhou, C., Martin, J. H., Jacobsen, A. J., Carter, W. B., \& Schaedler, T. A. (2016). Additive manufacturing of polymer-derived ceramics. Science, 351(6268), 58-62. doi:10.1126/science.aad2688

Falkingham, P. L. (2012). Acquisition of high resolution three-dimensional models using free, open-source, photogrammetric software. Palaeontologia Electronica, 15, 15.

Formlabs. (2017, March 28). The Ultimate Guide to Stereolithography: How SLA Works. Retrieved 22 September 2018, from https://www.youtube.com/watch?v=8a2xNaAkvLo

Frame, J., Lopez, N., Curet, O., \& Engeberg, E. D. (2018). Thrust force characterization of freeswimming soft robotic jellyfish. Bioinspiration \& Biomimetics, 13(6), 064001. doi:10.1088/1748-3190/aadcb3

Guo, N., \& Leu, M. C. (2013). Additive manufacturing: technology, applications and research needs. Frontiers of Mechanical Engineering, 8(3), 215-243.

Hull, C. W. (1986). Apparatus for production of three-dimensional objects by stereolithography.

Igic, B., Nunez, V., Voss, H. U., Croston, R., Aidala, Z., López, A. V., ... Hauber, M. E. (2015). Using 3D printed eggs to examine the egg-rejection behaviour of wild birds. PeerJ, 3, e965. doi:10.7717/peerj.965

Jones, R., Haufe, P., Sells, E., Iravani, P., Olliver, V., Palmer, C., \& Bowyer, A. (2011). RepRap the replicating rapid prototyper. Robotica, 29(1), 177-191. doi:10.1017/S026357471000069X

Joshi, S. C., \& Sheikh, A. A. (2015). 3D printing in aerospace and its long-term sustainability. Virtual and Physical Prototyping, 10(4), 175-185.

Klein, J., Stern, M., Franchin, G., Kayser, M., Inamura, C., Dave, S., ... Oxman, N. (2015). Additive Manufacturing of Optically Transparent Glass. 3D Printing and Additive Manufacturing, 2(3), 92-105. doi:10.1089/3dp.2015.0021

Laurentino, T. (2019, June 28). evALLution: 3 lessons the blind community taught us about inclusive teaching of evolution. Retrieved from https://ssecommunityblog.org/evallution-3-lessons-the-blind-community-taught-usabout-inclusive-teaching-of-evolution/

Lücking, T. H., Sambale, F., Beutel, S., \& Scheper, T. (2015). 3D-printed individual labware in biosciences by rapid prototyping: A proof of principle. Engineering in Life Sciences, 15(1), 51-56.

Lücking, T. H., Sambale, F., Schnaars, B., Bulnes-Abundis, D., Beutel, S., \& Scheper, T. (2015). 3D-printed individual labware in biosciences by rapid prototyping: In vitro biocompatibility and applications for eukaryotic cell cultures. Engineering in Life Sciences, 15(1), 57-64. doi:10.1002/elsc.201400094

Mitsopoulou, V., Michailidis, D., Theodorou, E., Isidorou, S., Roussiakis, S., Vasilopoulos, T., ... Theodorou, G. (2015). Digitizing, modelling and 3D printing of skeletal digital models of Palaeoloxodon tiliensis (Tilos, Dodecanese, Greece). Quaternary International, 379, 4-13. doi:10.1016/j.quaint.2015.06.068

Mota, C. (2011). The rise of personal fabrication (pp. 279-288). Presented at the Proceedings of the 8th ACM conference on Creativity and cognition, ACM. 
Murphy, S. V., \& Atala, A. (2014). 3D bioprinting of tissues and organs. Nature Biotechnology, 32(8), 773.

Niven, L., Steele, T. E., Finke, H., Gernat, T., \& Hublin, J.-J. (2009). Virtual skeletons: using a structured light scanner to create a 3D faunal comparative collection. Journal of Archaeological Science, 36(9), 2018-2023. doi:10.1016/j.jas.2009.05.021

O’Reilly-Pol, T., Kniepkamp, K., \& Johnson, S. L. (2017). Three-Dimensional Printed Fish Graders: A Tool to Rapidly and Reliably Size Select Zebrafish. Zebrafish, 14(3), 280-283. doi:10.1089/zeb.2016.1414

Oskui, S. M., Diamante, G., Liao, C., Shi, W., Gan, J., Schlenk, D., \& Grover, W. H. (2016). Assessing and Reducing the Toxicity of 3D-Printed Parts. Environmental Science \& Technology Letters, 3(1), 1-6. doi:10.1021/acs.estlett.5b00249

Parra-Cabrera, C., Achille, C., Kuhn, S., \& Ameloot, R. (2018). 3D printing in chemical engineering and catalytic technology: structured catalysts, mixers and reactors. Chemical Society Reviews, 47(1), 209-230.

Patra, S., \& Young, V. (2016). A review of 3D printing techniques and the future in biofabrication of bioprinted tissue. Cell Biochemistry and Biophysics, 74(2), 93-98.

Pearce, J. M. (2014). Open-source lab: how to build your own hardware and reduce research costs. Amsterdam: Elsevier.

Phillips, B. T., Becker, K. P., Kurumaya, S., Galloway, K. C., Whittredge, G., Vogt, D. M., ... Wood, R. J. (2018). A Dexterous, Glove-Based Teleoperable Low-Power Soft Robotic Arm for Delicate Deep-Sea Biological Exploration. Scientific Reports, 8(1), 14779. doi:10.1038/s41598-018-33138-y

Policha, T., Davis, A., Barnadas, M., Dentinger, B. T. M., Raguso, R. A., \& Roy, B. A. (2016). Disentangling visual and olfactory signals in mushroom-mimicking Dracula orchids using realistic three-dimensional printed flowers. New Phytologist, 210(3), 1058-1071. doi:10.1111/nph.13855

Prince, J. D. (2014). 3D Printing: An Industrial Revolution. Journal of Electronic Resources in Medical Libraries, 11(1), 39-45. doi:10.1080/15424065.2014.877247

Prusa, J. (2018, March 23). Photogrammetry - 3D scan with just your phone/camera. Retrieved 21 September 2018, from https://www.youtube.com/watch?v=ye-C-OOFsX8\&t=190s

Redwood, B., Schöffer, F., \& Garret, B. (2017). The 3D Printing Handbook: Technologies, design and applications (1st edition). Amsterdam: 3D Hubs.

Solid Concept. (2013a, May 15). Selective Laser Sintering (SLS) Technology. Retrieved 24 September 2018, from https://www.youtube.com/watch?v=9E5MfBAV_tA

Solid Concept. (2013b, June 3). Fused Deposition Modeling (FDM) Technology. Retrieved 24 September 2018, from https://www.youtube.com/watch?v=WH06G67GJbM

Tumbleston, J. R., Shirvanyants, D., Ermoshkin, N., Janusziewicz, R., Johnson, A. R., Kelly, D., ... DeSimone, J. M. (2015). Continuous liquid interface production of 3D objects. Science, 347(6228), 1349-1352. doi:10.1126/science.aaa2397

Vanderelst, D., Peremans, H., Razak, N. A., Verstraelen, E., \& Dimitriadis, G. (2015). The Aerodynamic Cost of Head Morphology in Bats: Maybe Not as Bad as It Seems. PLOS ONE, 10(3), e0118545. doi:10.1371/journal.pone.0118545 
Walker, M., \& Humphries, S. (2019). 3D Printing: Applications in evolution and ecology. Ecology and Evolution, 9(7), 4289-4301. doi:10.1002/ece3.5050

Watson, C. M., \& Francis, G. R. (2015). Three dimensional printing as an effective method of producing anatomically accurate models for studies in thermal ecology. Journal of Thermal Biology, 51, 42-46. doi:http://dx.doi.org/10.1016/j.jtherbio.2015.03.004

Weller, C., Kleer, R., \& Piller, F. T. (2015). Economic implications of 3D printing: Market structure models in light of additive manufacturing revisited. International Journal of Production Economics, 164, 43-56. 


\section{Appendices}

A zip archive (346 MB) containing all the files accompanying the appendices (referenced in the text below with double underline) can be downloaded from this link: https://uclouvainmy.sharepoint.com/:u:/g/personal/nicolas schtickzelle uclouvain be/EeJJ7nfXKxtKgOFakrdanwgBVGCbjEfEXN LjOgBqTrFLGw?e=AAgDCg

\begin{tabular}{|c|c|c|c|}
\hline $\begin{array}{l}\text { Name } \\
\text { [3D printing company] }\end{array}$ & URL & Type & $\begin{array}{l}\text { On- } \\
\text { demand } \\
\text { service }\end{array}$ \\
\hline CGTrader & www.cgtrader.com & Marketplace & $x$ \\
\hline Cults & cults3d.com/en & Marketplace & $\mathrm{x}$ \\
\hline $\begin{array}{l}\text { GrabCAD } \\
\text { [Stratasys] }\end{array}$ & grabcad.com & Repository & \\
\hline MyMiniFactory & www.myminifactory.com & Marketplace & \\
\hline NIH & 3dprint.nih.gov & Repository & \\
\hline $\begin{array}{l}\text { Pinshape } \\
\text { [Formlabs] }\end{array}$ & pinshape.com & Marketplace & \\
\hline Repables & repables.com & Repository & \\
\hline Redpah & www.redpah.com & Marketplace & \\
\hline Shapeways & www.shapeways.com & Marketplace & $x$ \\
\hline STLFinder & www.stlfinder.com & Search engine & \\
\hline $\begin{array}{l}\text { Thingiverse } \\
\text { [Makerbot] }\end{array}$ & www.thingiverse.com & Repository & \\
\hline Threeding & www.threeding.com & Marketplace & \\
\hline Trinpy & www.trinpy.com & Marketplace & \\
\hline Yeggi & www.yeggi.com & Search engine & \\
\hline $\begin{array}{l}\text { YouMagine } \\
\text { [Ultimaker] }\end{array}$ & www.youmagine.com & Repository & \\
\hline
\end{tabular}

Table S1. Major online sources of digital 3D model files, with the commercial 3D printing company they are linked to where appropriate. Models can be downloaded for free from repositories, whereas marketplaces host models for buying (some may be free too); some sites also offer an on-demand service where the user can request the digital 3D model being specifically created for a fee. Search engines do not host digital 3D model files but allow specific search for them on the internet. Due to the highly dynamic nature of the 3D printing community, this list should not be considered exhaustive or reflecting any quality ranking. 


\begin{tabular}{|l|l|}
\hline Company name & URL \\
\hline 3D Hubs & $\underline{\text { www.3dhubs.com }}$ \\
\hline FacFox & $\underline{\text { facfox.com }}$ \\
\hline iMakr & $\underline{\text { www.imakr.com }}$ \\
\hline i.materialise & $\underline{\text { i.materialise.com/en }}$ \\
\hline Kraftwurx & $\underline{\text { www.kraftwurx.com }}$ \\
\hline Makexyz & $\underline{\text { www.makexyz.com }}$ \\
\hline Materialise OnSite & $\underline{\text { onsite.materialise.com }}$ \\
\hline Meltwerk & $\underline{\text { www.meltwerk.com/en }}$ \\
\hline MyMiniFactory & $\underline{\text { www.myminifactory.com }}$ \\
\hline Protolabs & $\underline{\text { www.protolabs.co.uk }}$ \\
\hline Quickparts & $\underline{\text { www.3dsystems.com/quickparts }}$ \\
\hline Sculpteo & $\underline{\text { www.sculpteo.com/en }}$ \\
\hline Shapeways & $\underline{\text { www.shapeways.com }}$ \\
\hline Stratasys direct & $\underline{\text { www.stratasysdirect.com }}$ \\
\hline Trinckle & $\underline{\text { www.trinckle.com/en }}$ \\
\hline Unic-3D & $\underline{\text { unic-3d.com/en }}$ \\
\hline Xometry & $\underline{\text { www.xometry.com }}$ \\
\hline
\end{tabular}

Table S2. Major 3D printing professional services. Not all companies ship worldwide. Some websites (e.g. All3DP, https://all3dp.com/) offer a price comparison service: once a digital 3D model file is uploaded and the printing technology and material chosen, a quote from several companies will be displayed. Due to the highly dynamic nature of the 3D printing community, this list should not be considered exhaustive or reflecting any quality ranking. 


\section{1) Making a digital 3D model of a bunch of hazelnuts using photogrammetry}

To illustrate how an existing object can be scanned into a digital 3D model, we chose a bunch of hazelnuts. It presents a relatively complex 3D shape, with concave and convex parts, as well as a relatively uniform colour, features that make it not the easiest object to scan.

We used photogrammetry to create a digital 3D model of it, using a simple setup that should be accessible to any scientist:

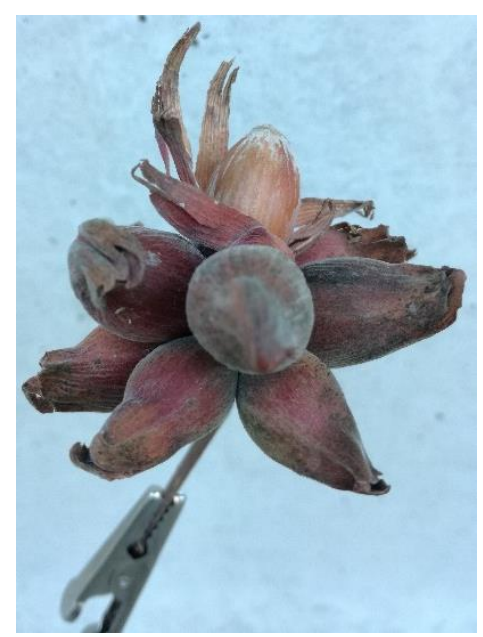
we placed the object onto a support, shot a series of 96 pictures of it from different angles covering all its faces using a smartphone, and treated the pictures using the free education license of Autodesk Recap Photo (https://www.autodesk.com/education/home). It is likely that some post-processing treatment will be necessary to crop the object when some parts of the background are kept in the digital 3D model, or to correct small errors. However, as the example here shows, we found the results to be very good even if the setup to shoot the pictures was not optimized.

Step 1: setup the object to be able to shoot pictures of it covering all its faces with light as uniform as possible. We found that placing it on some elevated support (such as a tripod) and shooting outside gives best results. Indeed, having a heterogeneous background some distance behind the object offers reference points to the photogrammetry analysis that will move much more between pictures, helping to discriminate the object from the background and computing the position of the camera in space.

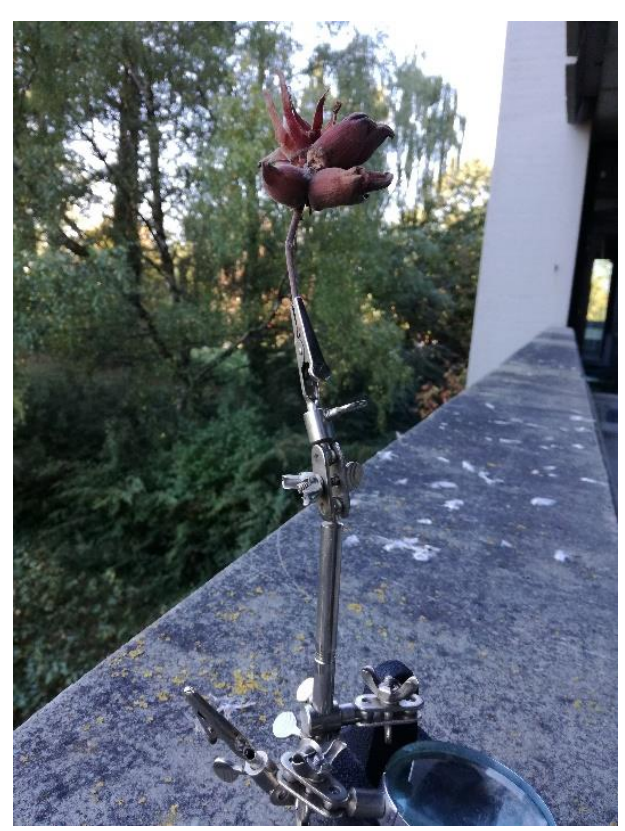

Step 2: shoot pictures of the object from various angles and covering all its faces, ensuring a large overlap between pictures. More pictures are better than fewer. Also try to keep the camera parameters as constant as possible, by deactivating the flash and if possible shooting under manual mode with fixed shutter speed and aperture. However, the example shown here was on purpose suboptimal (Huawei P9 Lite smartphone with automatic shooting parameters except flash was deactivated) to show how the result can be with a minimal setup; shooting the object with a camera and fixed parameters should have given an even better result. [These $\underline{\underline{96 \text { pictures }}}$ are provided in the accompanying files to explore by yourself the creation of this digital 3D model using photogrammetry.] 


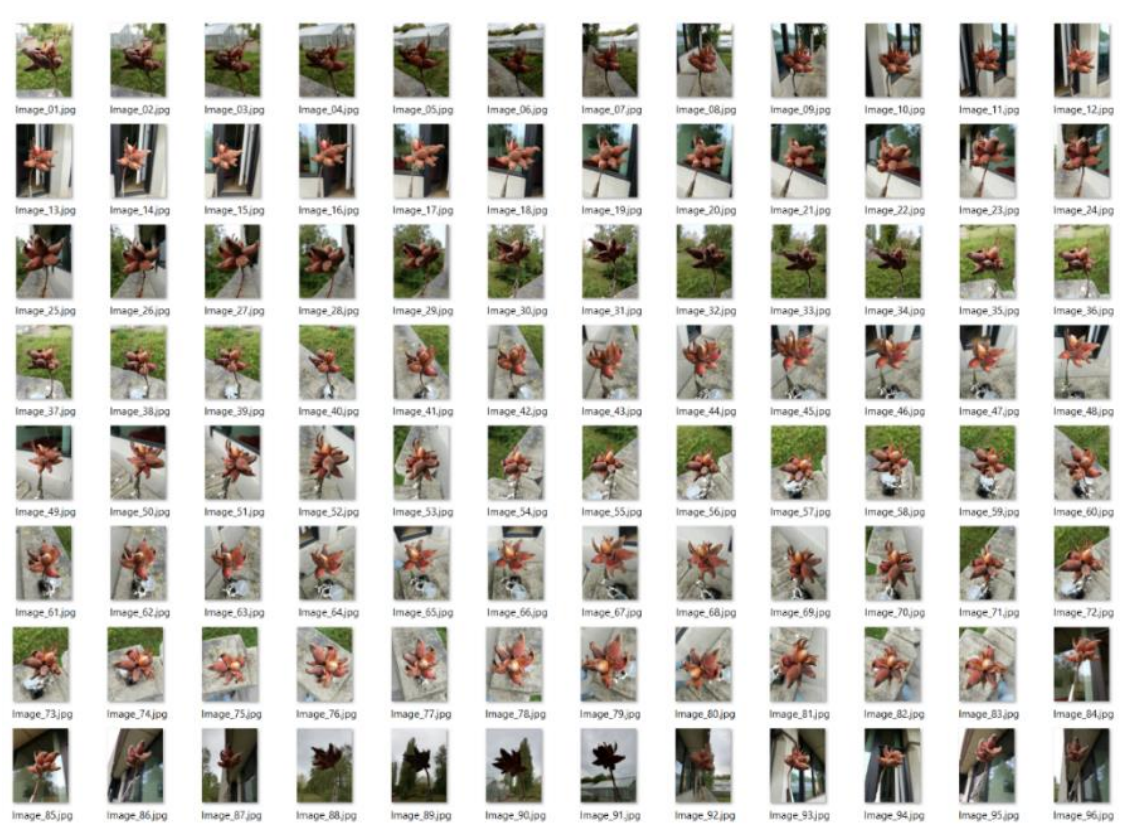

Step 3: create the digital 3D model with Autodesk Recap Photo: run "create 3D $\backslash$ Object", load the pictures and let the analysis run in the cloud.

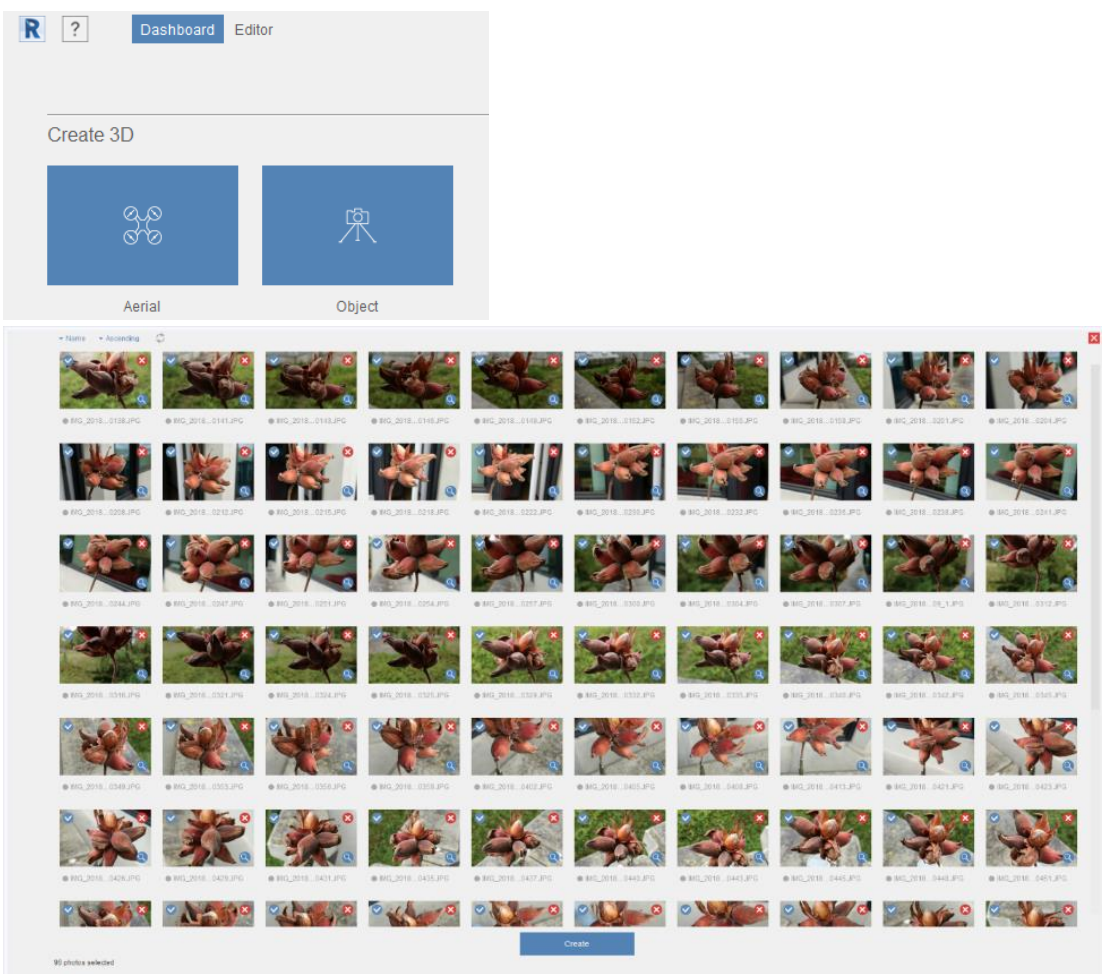

The free education license does not have a high priority rank for analysis in the cloud (Autodesk prioritizes paid uses), so the analysis can take a few hours.

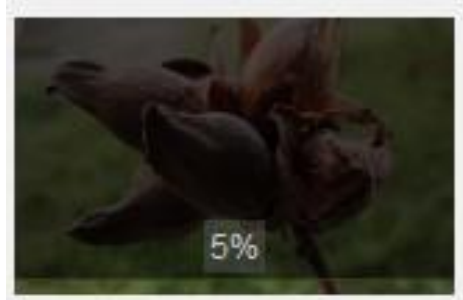

Uploading hazelnuts-1

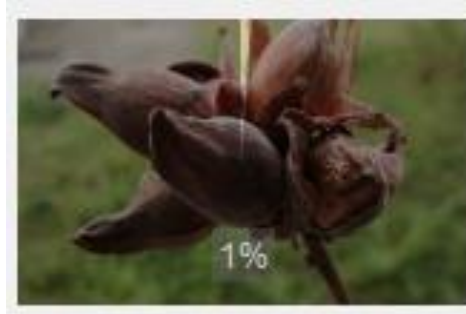

Waiting in queue... 
Step 4: when the analysis is finished, download the model, display it and perform post-processing steps if needed. Here a part of the support was included in the model, so we cropped it.

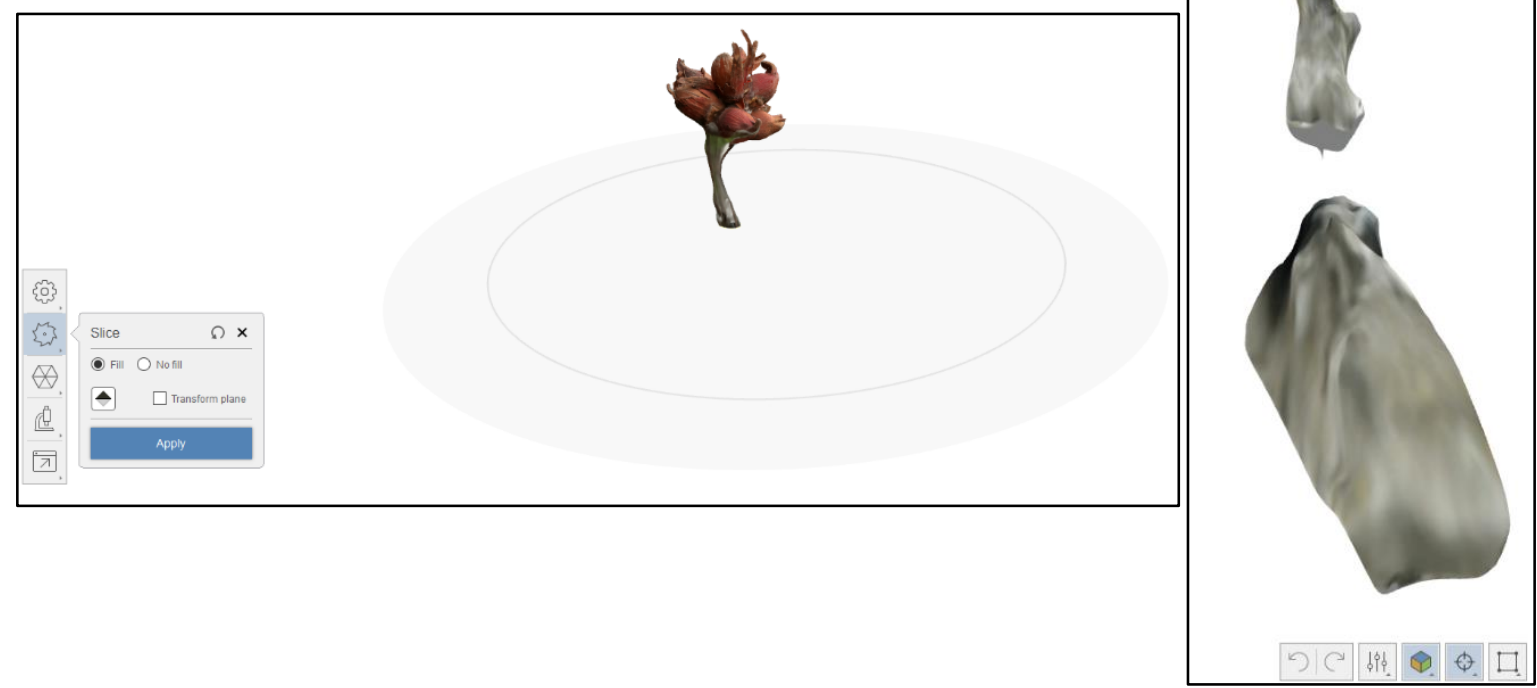

The end result is very good; you can explore it by yourself by opening the "Hazelnut Photogrammetry - digital 3D model.stl" file in any 3D file viewer or directly the result file of Autocad Recap photo ("Hazelnut Photogrammetry - digital 3D model.rcm" file). Errors are mainly located on the underside, where some background was integrated into the object because it was not heterogeneous enough; it appears in green in the final digital 3D model and corresponds to some vegetation visible in the background. If this is a problem, either correct the digital 3D model with post-processing or modify the shooting location to increase background heterogeneity and reshoot a series of pictures.
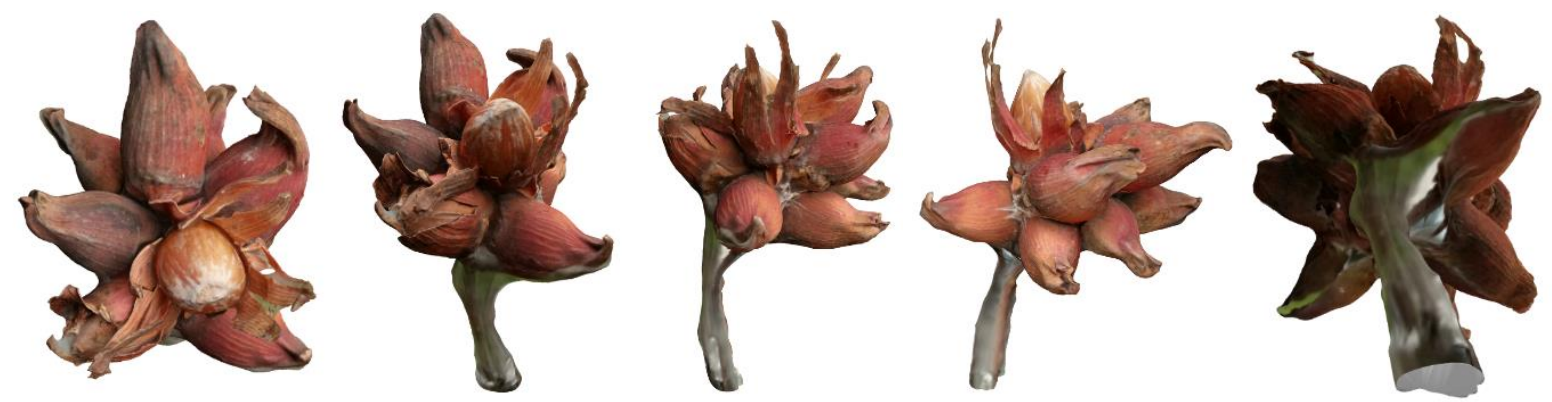


\section{2) Creating a microtube rotor for a mini centrifuge using 3D builder drawing software}

To create the microtube rotor for a mini centrifuge (main text, Fig. 20) using a drawing software, we used 3D builder, a free software available in Microsoft store. It took around one hour to create this object, without prior knowledge of the 3D builder software. This is then a very cost-effective approach if one needs to create a simple geometric object. However, and contrary to the same object created with a parametric code software (see next section), the object must be redrawn from scratch if it needs to be modified, even slightly, (e.g. to accommodate four tubes instead of six) and may present small errors (e.g. in step 8 the spokes were not perfectly aligned vertically). Saving intermediary designs might make creating variants easier but the parametric based approach will also make this faster as is illustrated in the next section. Below we present the 11 steps needed to create the digital 3D model ("Centrifuge rotor - drawing software.3mf" file) of this very simple geometric object.

Step 1: create a cylinder (diameter $=60 \mathrm{~mm}$, height $=10 \mathrm{~mm}$ ).

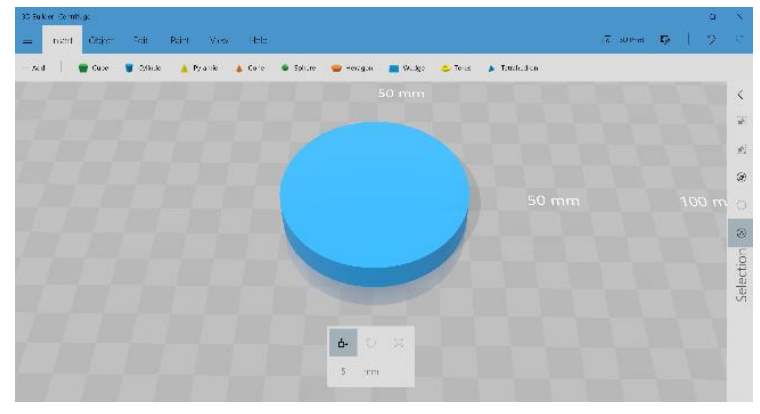

Step 2: create another cylinder (diameter $=50$ $\mathrm{mm}$, height $=20 \mathrm{~mm}$ ), with a smaller diameter and the same centroid meant to create a hole in the outer cylinder and create the rotor rim.

Note how it is good practice in such a case to

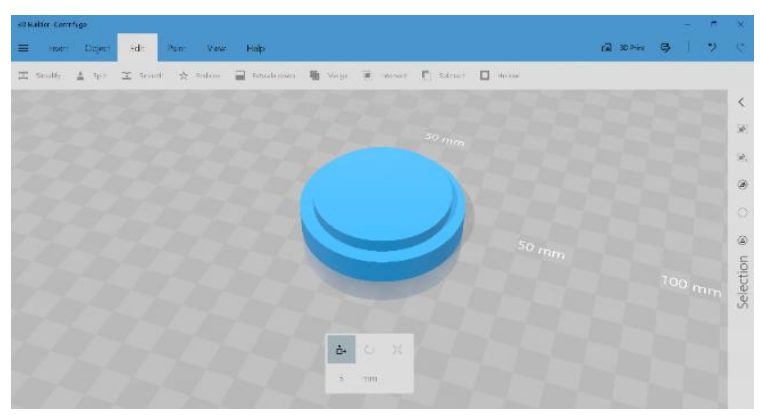
make the shape to subtract (negative shape) a bit larger (here higher) than the positive shape to avoid problems in the object definition when faces of the two shapes (the positive and the negative) perfectly coincide. 
Step 3: select the inner cylinder and use "edit\subtract" command to create a hole in the outer cylinder having the shape of the inner cylinder.

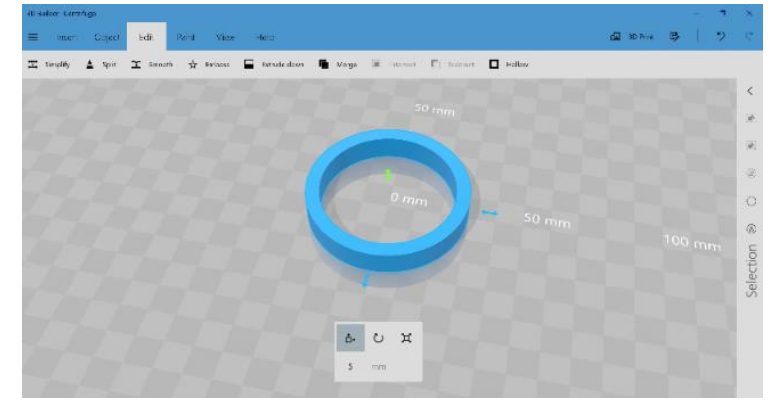

Step 4: create a cylinder (diameter $=8 \mathrm{~mm}$, length $=65 \mathrm{~mm}$ ), rotate it; then create a cube (section $=8 * 8 \mathrm{~mm}$, length $=65 \mathrm{~mm}$ ) partly overlapping the cylinder. Select both shapes and use "edit\merge" command to merge them into a single shape.

Step 5: move this shape below so it overlaps with the rotor rim where the holes for the microtubes should be.
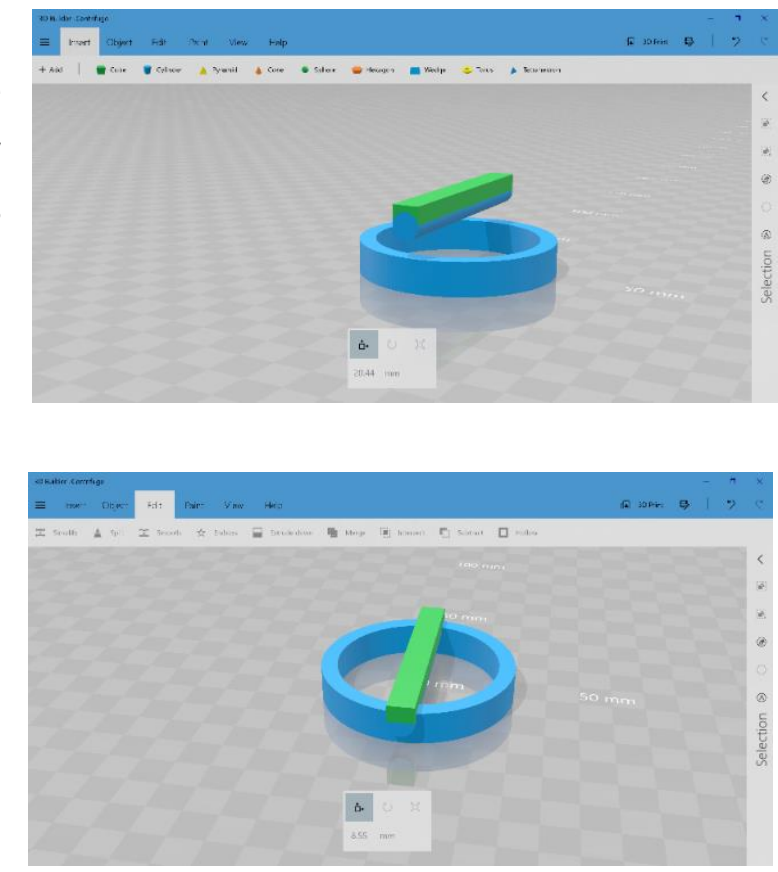

Step 6: duplicate this shape twice, rotate them and place them at the appropriate places where the six holes for the microtubes must be created.

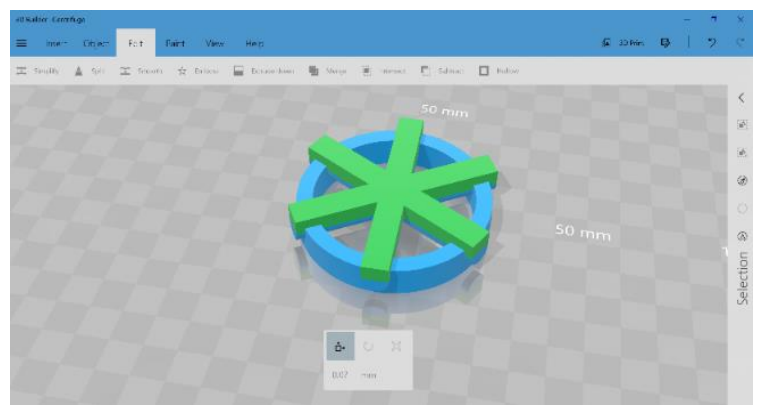


Step 7: select the three shapes, use "edit\merge" command to merge them into a single shape, then use "edit\subtract" command to create the six holes for the microtubes.

Step 8: create a cube (section $=5 * 5 \mathrm{~mm}$, length $=60 \mathrm{~mm}$ ), duplicate it twice, rotate and move them to create the spokes inside the rotor rim.

Step 9: create another cylinder (diameter $=5$ $\mathrm{mm}$, height $>5 \mathrm{~mm}$ ), with the same centroid meant to create a central hole to attach the microtube rotor to a drill.
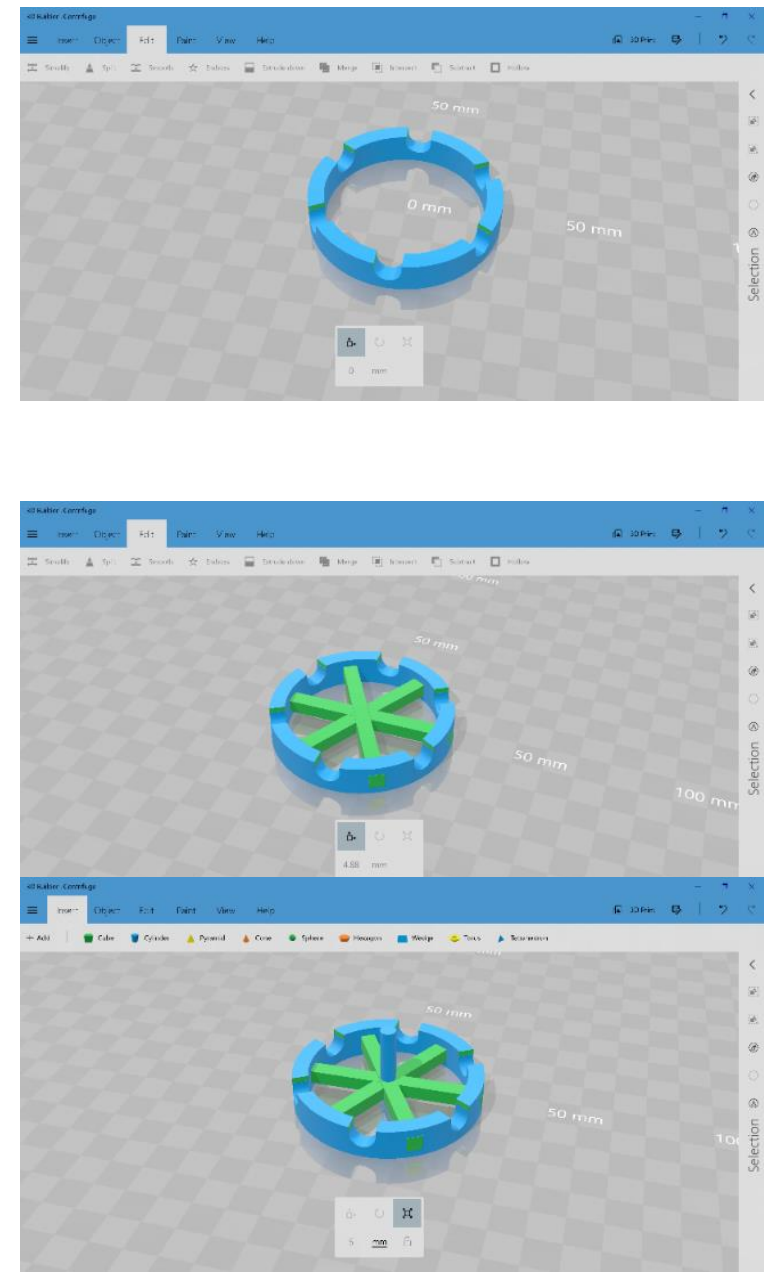

Step 10: select the cylinder and use "edit\subtract" command to create the hole.

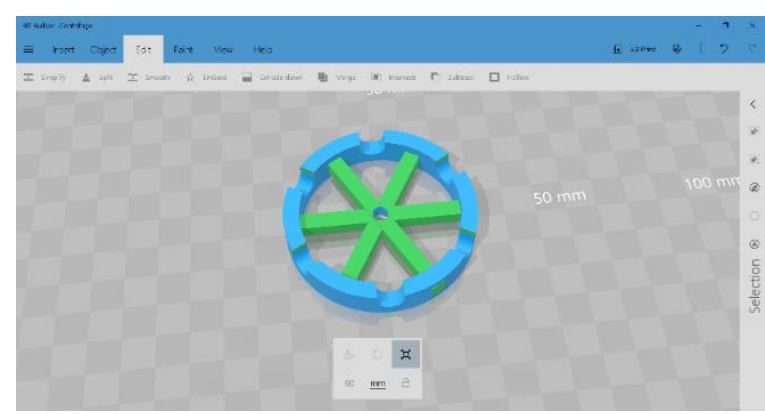

Step 11: use the "save as \STL format" command to export the digital 3D model in the stl format, to be sent to the 3D printer.

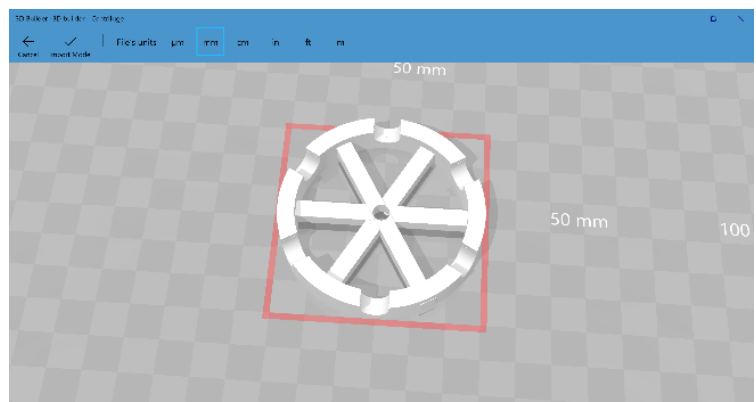




\section{3) Creating a microtube rotor for a mini centrifuge using a parametric code software}

To create the microtube rotor for a mini centrifuge (main text, Fig. 20) using a parametric code software, we used OpenSCAD, a free, open source and mulit-platform software available at www.openscad.org. It took around one hour to create this object, for someone with a considerable prior knowledge of writing scripts for OpenSCAD. This approach is best when one wants to be able to create variants of the same objects very easily, simply by adjusting some parameters in the script. Below we present the script ("Centrifuge rotor - parametric code.scad" file) used to create the digital 3D model of this very simple geometric object, which is divided into two main sections: (1) a list of input values describing a set of parameters (here various measures about the dimensions of the object and the number of microtubes handled by the rotor), and (2) the script rendering the object based on those input values.

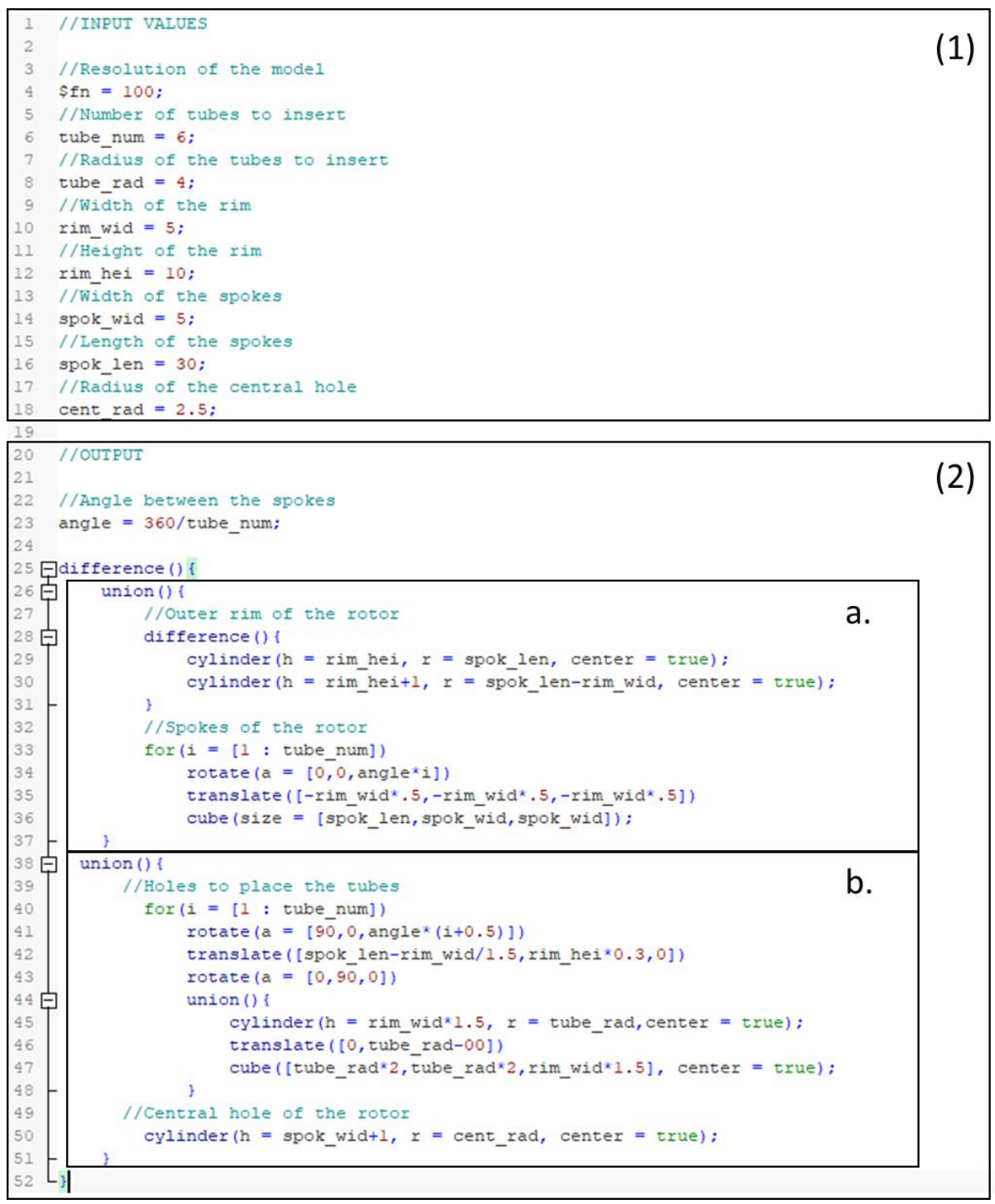


This simple model is produced by using the difference function of OpenSCAD, which removes a specific set of parts from an initial object. The "output" section of the script (2) describes the "initial object" (a), which is made up of the rotor rim and its spokes. From this object, we removed the parts corresponding to the holes for the microtubes and the central hole to attach the rotor to the drill (b) to create the final object (c) (see illustration below).

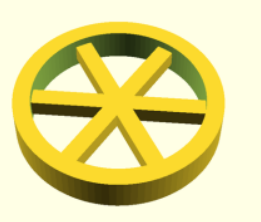

b.

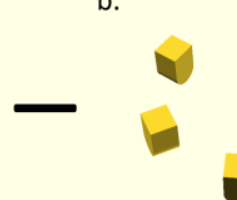

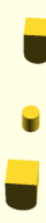

c.

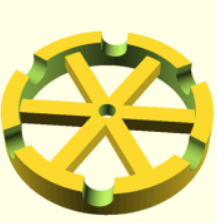

Note that in OpenSCAD, it is not possible to hollow a shape created in a previous step; this makes it necessary to first hollow the rim (using the difference function, lines 28-31), then add the spokes (lines 32-36), and only after that subtract the holes to place the microtubes and attach the drill. There are alternative scripts that will create the same object; e.g. it is possible to create the hollow rim with holes for the microtubes in one step, and add the spokes with the central attachment hole in another step. OpenSCAD language is very powerful and integrates the use of macros (called modules), which allows creating very complex digital 3D models relatively easily once mastered. 


\section{4) Pre-processing steps: what to consider to optimize print quality}

\section{Adding supports}

During the printing process, the object may be submitted to several forces (gravity, peeling process, temperature and/or curing stresses) and their actions need to be counterbalanced to keep the object integrity. This mainly involves adding support structures to the object. Such supports will often have a negative impact on surface finish, may be hard and/or long to detach from the object, and consume material; optimizing their number and position is therefore highly desired. With material extrusion printing techniques, supports must be added for all structures that overhang over empty space (e.g. a bridge, or the ceiling of a hole in an object) as soon as the angle is large $\left(45^{\circ}\right.$ can be used as a rule-of-thumb but varies with material and printer) to avoid collapse due to gravity before the material is sufficiently solidified. Top-down photopolymerization similarly requires support for overhanging features. In bottom-up photopolymerization, objects are also subject to gravity but more importantly they are subjects to forces due to the peeling process, where the newly formed layer must be detached from the bottom of the resin vat. This peeling process creates forces that are potentially very important if the area of the layer is large. Supports are then needed to attach the object, and especially overhanging structures to the build platform (external supports) or to other parts of the object (internal supports). In powder-based technologies, unmelted powder acts as a support for the object as it is produced. No additional support is then needed, which gives additional freedom in the object design, saves material and allows more objects to be printed at once because they can be freely placed into the whole build volume. The software accompanying many commercial printers can usually add supports automatically according to the object shape and printing orientation. But manual adjustments may be needed to optimize them; our personal experience show that supports can often be reduced to save post-processing work and material because supports added by the printer software are usually overestimated to ensure correct printing. If not performed automatically by the printer software at time of printing, supports must be integrated as extra shapes in the digital 3D model file. 


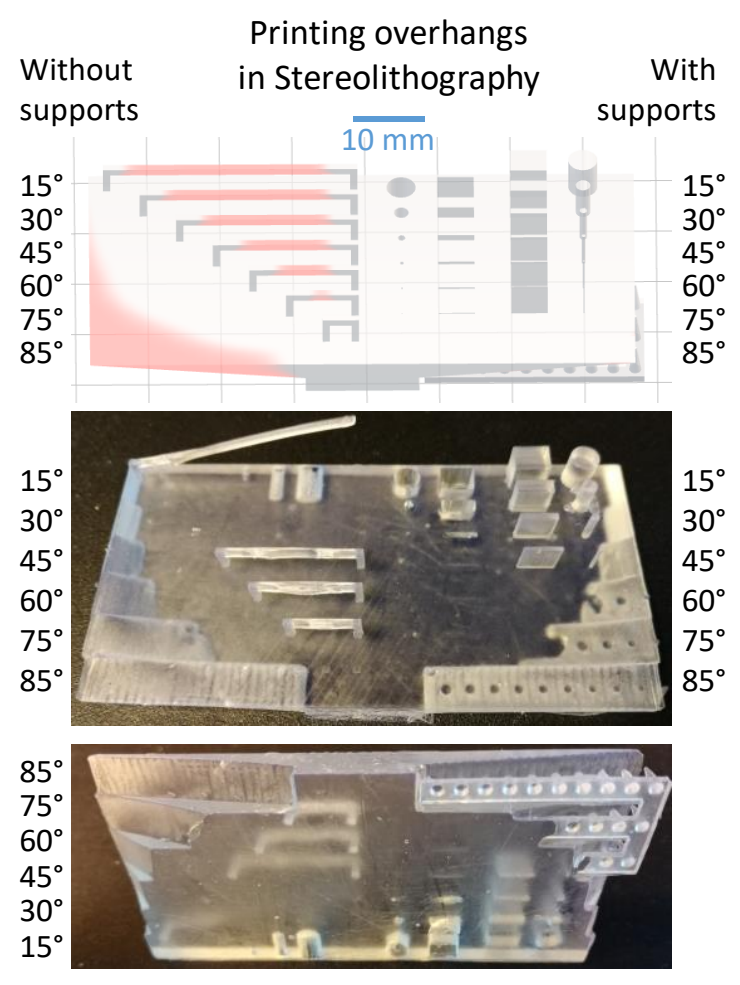

To illustrate the impact of supports for printing overhanging structures, we created a composite object called the "features" part ("Preprocessing display - features.stl" file), which presents a series of seven bridges ( $1 * 1 \mathrm{~mm}$ cross section, $5 \mathrm{~mm}$ high, 5 to $35 \mathrm{~mm}$ long), and a series of 6 overhangs $\left(15,30,45,60,75\right.$ and $85^{\circ}$ angles) without supports on the left side, and with supports added on the right side (custom supports integrated into the digital 3D model). Here, we printed it using SLA on a Form1+ printer (www.formlabs.com) with clear resin (v2), a layer thickness equalling $0.1 \mathrm{~mm}$, and placed flat on the build platform with no extra supports added via the printer software. Printing time was $50 \mathrm{~min}$ and used $12 \mathrm{ml}$ of clear resin. On the top picture, note the areas (marked in red) where the printer software expects problematic printing due to unsupported overhangs. The central and bottom pictures show that the printed part indeed presented some structural failures, but on some of these problematic areas only, mainly the bridges. [Note that some very small features may sporadically fail to print in SLA because they stay attached to the bottom of the vat instead of the build platform during on peeling step; this was the case for the $5 \mathrm{~mm}$ bridge, which should print correctly another time because of its small overhang but its pillars failed to print this time.] All the overhangs at an angle printed nearly perfectly, with no visible impact of adding supports; this illustrates that the printer software is on the safe side when adding support for overhangs and that SLA is often very good at printing structures that overhang at an angle; trial and errors would have allowed to remove the supports for these overhangs, saving time in postprocessing and ensuring a smoother finish. 


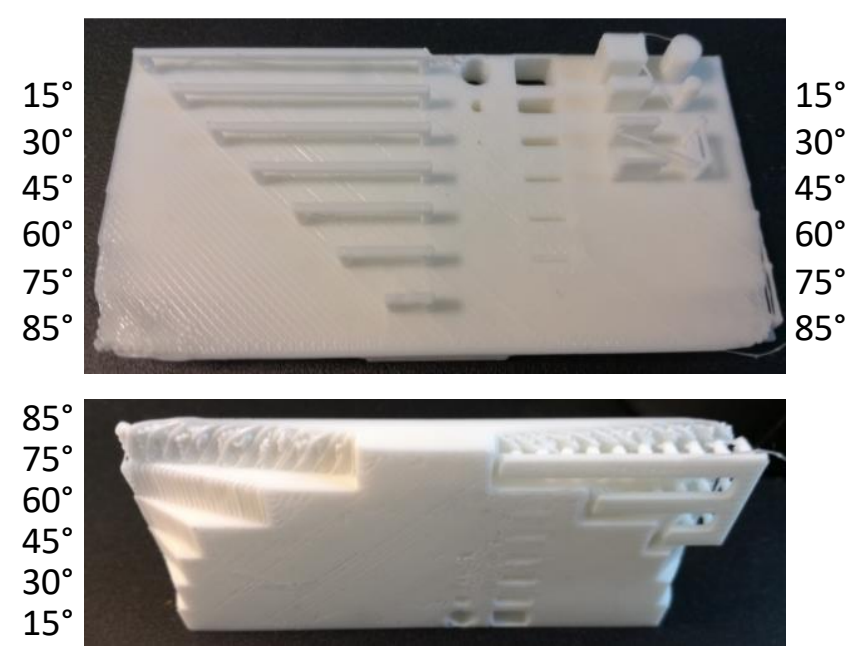

The same "features" object printed at Makilab (Fablab existing in our university city: https://makilab.org) using FDM on a Sigma BCN3D printer with white PLA filament, a layer thickness equalling $0.2 \mathrm{~mm}$, and placed flat on the build platform with no extra supports added via the printer software. The stl file was sliced using Cura software and sent to the printer as a gcode file. Printing time was $56 \mathrm{~min}$ and used $9 \mathrm{~g}$ of PLA filament. As with the SLA printing, overhangs and bridges printed surprisingly well despite the absence of supports. Even the $85^{\circ}$ overhang printed nearly completely, although its inferior surface shows a very poor quality (as shown on the bottom picture).

\section{Printing enclosed volumes}

Printing objects comprising enclosed volumes needs a special note. Apart from potential internal supports required for printing the ceiling layer, enclosed volumes printed with photopolymerization and powder-based technologies need one or several holes placed somewhere in the external wall of the volume. The first reason is they serve as escape holes to allow the uncured resin or unmelted powder to be extracted from the object after printing is finished. In bottom-up photopolymerization, such holes also serve to relieve the negative pressure that will be created inside the enclosed volume by suction forces during the peeling process, i.e. the action of separating the newly cured layer of the bottom of the resin vat. Such suction forces can be strong enough to break the walls of the enclosed volume if they are too thin, as the pictures below displays. This was a very frequent sources of printing problem in the early days of bottom-up photopolymerization with consumer printers (such as Formlabs Form 1+) because of failure of users to understand this physical force before it was made explicit in the documentation. These holes may need a post-processing step where they are sealed. 


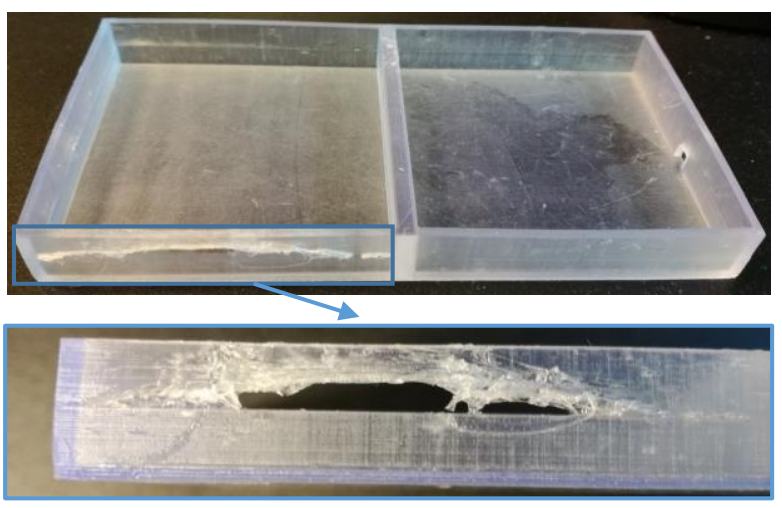

The "enclosed volumes" part (printed using SLA Form1+ printer using clear resin v2, a layer thickness equalling $0.1 \mathrm{~mm}$, and placed flat on the build platform with no extra supports added via the printer software) illustrates how suction forces during the peeling process make an enclosed volume (left volume) to implode, and its front wall to break at some point. Adding a hole $\left(3^{*} 3 \mathrm{~mm}\right.$ in the right wall of the right volume) allows pressure to equalize during the peeling process, avoiding the breaking of the wall. Note that the volume is open on the top but this side is attached to the bottom of the vat when printing, making the volume effectively enclosed and subject to suction forces.

For printing full volumes -and not empty enclosed volumes-, photopolymerization and powder-bed techniques simply solidify the whole volume of resin/powder, while material extrusion technologies can fill the volume with a more or less dense infill (a 3D mesh of walls, see pictures below) to increase print speed and decrease the amount of material used without sacrificing the structural properties of the object (e.g. resistance against compression). For an illustration of more complex mesh patterns, see e.g. https://www.youtube.com/watch?v=VRorEe1Ka5I.

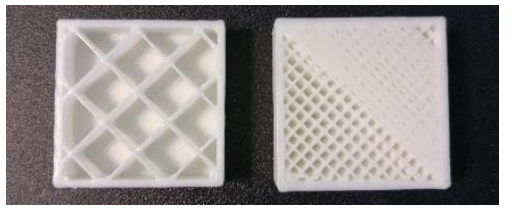

A cube printed using FDM on a Sigma BCN3D printer with white PLA filament, showing two values for the infill (15\% on left, 30 on right). Note that both prints were stopped before finishing in order to keep the interior visible; on the right picture, the first full layer of the cover has been started, covering the top right half of the cube. 


\section{Orienting the object for printing}

The fact that 3D printing technologies print the object one layer at a time implies that: (1) the object is anisotropic, with material cohesion being better within a layer than among layers (2) the resolution in the $\mathrm{Z}$ axis (vertical) differs from (and is often lower than) resolution in $\mathrm{X}$ and $Y$ axes (horizontal), especially in FDM where the thickness of a layer can be up to $400 \mu \mathrm{m}$; and (3) the object is subject to shrinkage and wrapping because of the stresses applied to the material as part of the printing process: temperature stress in FFF and SLS, where the material will cool down and shrink after being heated during printing; curing stress in photopolymerization and material jetting, where curling can occur when a layer shrinks on top of a previously solidified layer. It is then best to keep this in mind when orienting the object for printing, e.g. by placing the finer details on the horizontal plane, printing a large flat section vertically or at an angle (not horizontally) to limit curling and the forces linking the cured layer to the vat, or adding supports among parts of the object and/or to the build platform to mitigate the risk of wrapping and curling.

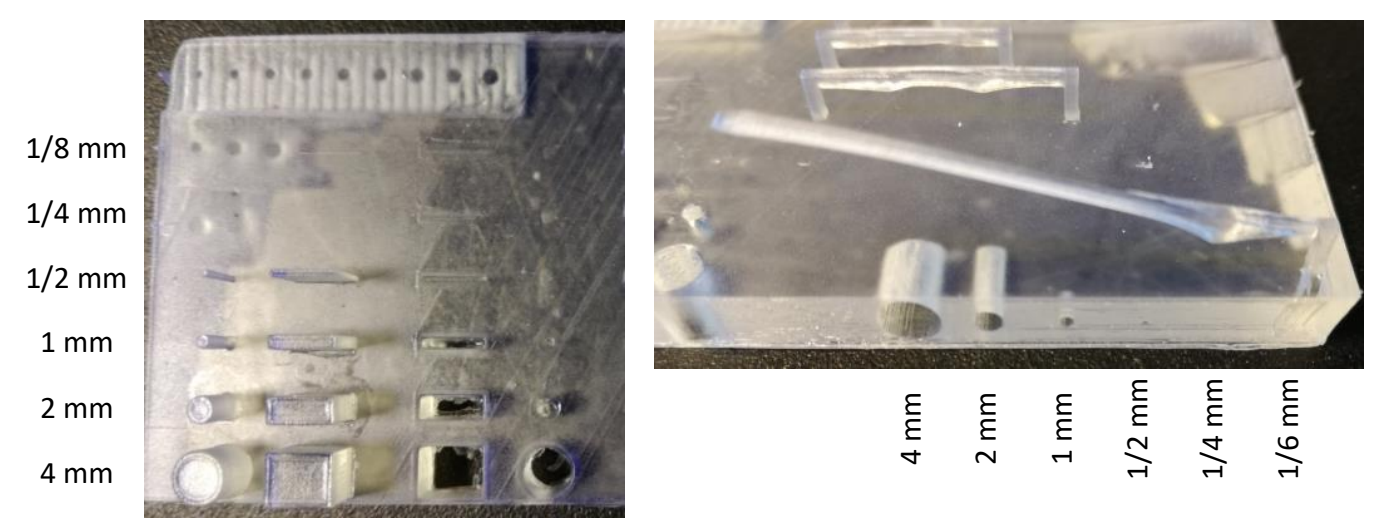

As an example of the impact of object orientation on print, we show here (using the same "features" part presented above) how a $2 \mathrm{~mm}$ wide hole failed to print vertically (left picture) because forces squeeze each printed layer between the build platform and the bottom of the vat, making it to expand horizontally and fill the hole. The same hole printed successfully when oriented horizontally (right picture). Note that this stereolithography printer perfectly prints positive features (walls and pins) down to a $0.5 \mathrm{~mm}$ size, but negative features (holes) are obstructed, at least partially, already at $2 \mathrm{~mm}$ when printed vertically and at $1 \mathrm{~mm}$ when printed horizontally because layers are squeezed horizontally. 
This is the printing orientation chosen and the supports added by the Preform software accompanying our Form1+ stereolithography printer when choosing the automatic mode for printing the "features parts". Note how it differs from the "flat printing on the build platform" approach we chose. This orientation is optimized to print the part features by minimizing overhangs and the printing of large areas on the same layer, but removing support marks is going to be a painful process and will leave marks on the object. This illustrates how choosing printing orientation may have important consequences for the final quality of the printed object and the post-processing work required.

Printing the object in several parts independently, each with its best orientation, and assembling them afterwards can be a very efficient solution, especially when conflicting orientation guidelines appear among parts of the object or when the object is larger than the printer's build volume (https://formlabs.com/blog/how-to-create-models-larger-than-your3d-printers-build-volume/). Objects printed in stereolithography can be easily assembled by gluing them with the same photopolymer resin they are built from, because it can be manually cured with the appropriate light (e.g. $405 \mathrm{~nm}$ violet light for Formlabs resin), a process that can also be used to seal pressure relieving holes in the walls of enclosed volumes. Objects printed in ABS can be glued together using cold welding with acetone. 


\section{5) Sending the digital 3D model to the printer}

On professional and ready-to-use consumer printers, the digital 3D model can often be imported directly into the printer software (often in the .stl format), which can take care of some of the pre-processing steps, such as best orienting it and adding supports where needed, as explained before. Such software can also take care of two additional specific steps needed for printing: model checking and slicing. However, when using some homemade and RepRap printers, the user may need to perform these steps manually before the model can be sent to the printer. The first step is to check the 3D digital model for errors in the polygonal mesh, such as holes (the model is not watertight) that can lead to software error when printing. Models generated by 3D scanners or photogrammetry apps are particularly error prone, while models generated by CAD are less, especially when good practices are followed during the design. Various online and offline, free or paid, software exist to check and repair model (a review can be found at all3dp.com/1/stl-repair-stl-file-online-checker-fix-3d-model/). The second step is to slice the 3D object into 2D layers because 3D printers, whatever the technology, print the object as a succession of layers one above (or under) the other. Here also, many tools exist to perform the slicing (a review can be found at all $3 \mathrm{dp}$.com/1/best-3dslicer-software-3d-printer/); searching the internet with "3D print slicer" will also work (be sure to specify "print" because "3D slicer" is a software for image visualization in the scientific and medical context). 


\section{6) Post-processing the printed object}

Once the 3D printing of the object is finished, the object is rarely ready for use. Some postprocessing is needed, depending on the printing technology and the final use of the object. Here we will outline the most frequent post-processing steps. First, in photopolymerization and powder-based technologies, the object must be separated from uncured/unmelted material. Uncured resin is usually washed off by rinsing the object in IPA (Isopropyl alcohol or isopropanol), while unmelted powder can be removed by brushing or vacuum aspiration. Specific actions may be needed to empty enclosed volumes, such as actively rinsing them by forcing isopropanol into them (e.g. using a syringe). Second, supports added to the object to deal with forces during the printing process need to be removed. Some printers allow printing the supports in another, dissolvable material, making the removal of support easily performed by rinsing the object into a chemical solution (https://www.youtube.com/watch?v=0ENgGkPP94w). But the process can also involve physically cutting the supports at their attachment point on the object if supports are made of the same material as the object, such as in basic FDM or photopolymerization. This can be long and difficult, especially in complex objects or when internal supports are used. Also, marks will remain visible at these attachment points and some surface treatment (e.g. polishing) may be needed. This makes the optimizing of support number and position a key pre-processing step. Third, some material need extra treatment before they reach their final mechanical properties; e.g. many photopolymer resins need some post-curing with UV light (https://formlabs.com/blog/introduction-post-curing-sla-3d-prints/).

Besides these compulsory post-processing steps, optional actions can change the appearance or mechanical properties of the object. The variety of such treatments is too large to offer a complete review here, but we illustrate a few ideas. Placing objects printed in ABS in an atmosphere with acetone vapours will dissolve the object surface, creating a shiny smooth surface without the striated aspects due to visible layer limits (https://www.youtube.com/watch?v=6xFUNFG-UKE). The same can be obtained on a larger range of materials ( $A B S$, PLA, photopolymerization resin...) by treating the surface with a product such as XTC-3D (https://www.smooth-on.com/products/xtc-3d/). Polishing and varnishing objects printed in clear photopolymer resin can render them fully transparent (https://support.formlabs.com/s/article/Polishing-Clear-Resin-for-a-Transparent-Finish).

Many materials also allow painting to change the surface colour or finish. Finally, techniques have been developed to cover 3D printed objects with a layer of metal, a process known as electroplating (formlabs.com/blog/combining-3D-printing-and-electroplating-for-replicableexperimentation). 